\title{
Correlation between morphology, rheological behavior and electrical behavior of conductive co-continuous LLDPE/EVA blends containing commercial graphene nanoplatelets
}

\author{
Emna Helal ${ }^{\mathrm{a}, \mathrm{b}}$, Rafael S. Kurusu ${ }^{\mathrm{a}, \mathrm{b}}$, Nima Moghimian ${ }^{\mathrm{b}}$, Giovanna Gutierrez ${ }^{\mathrm{b}}$, Eric David ${ }^{\mathrm{a}}$, \\ Nicole R. Demarquette ${ }^{a *}$ \\ *Corresponding author: nicoler.demarquette@etsmtl.ca \\ ${ }^{a}$ Mechanical Engineering Department, École de Technologie Supérieure - ÉTS, 1100 Notre-Dame Street West, \\ Montréal, Québec - Canada H3C 1K3 \\ bNanoXplore Inc., Saint-Laurent (Montréal), QC, H4N 2G3, Canada
}

\begin{abstract}
In this work, co-continuous blends of linear low density polyethylene (LLDPE)/ Ethyl vinyl acetate (EVA) containing graphene (GN) have been studied. Although mass-produced GN grade prepared by mechanochemical exfoliation of graphite and a facile melt compounding technique were adopted, it was possible to lower the electrical percolation threshold significantly by controlling the localization of GN nanoplatelets in the blend and by applying an appropriate thermal annealing procedure. The electrical and rheological properties of the obtained nanocomposites were systematically investigated to get an insight on the composite morphology. During annealing, an alignment between time-dependent behaviors of viscoelastic moduli and electrical conductivity was observed. An increase of both quantities and a simultaneous coarsening of the blend's morphologies occurred during the first 30 minutes of annealing followed by a more stable behavior. This rise was attributed to the diffusion and flocculation of GN nanoplatelets and their migration to the interface. Furthermore, the electrical and rheological percolation threshold concentrations were evaluated using a scaling Power law. The electrical percolation threshold was reduced to 0.5 vol\% upon thermal annealing and was close to the rheological percolation threshold. Finally, the viscoelastic response of the composites was well described by a two-phase model, indicating that the effect of the relaxation dynamics of the interfacial network does not depend on the blend's morphology, even though the latter affects the space arrangement of GN and consequently the strength of the formed network.
\end{abstract}

Key words: graphene, co-continuous blends, interfaces, thermal annealing, percolation threshold, viscoelastic behavior, two-phase model.

\section{Introduction}

Electrically conductive and semi-conductive polymer composites are being more and more involved in a wide range of industrial applications including flexible electronics, soft robotics, health monitoring devices, anti-static materials, sensors, protective screens for power cables, electric field-grading materials for cable accessories and electromagnetic interference shielding materials [1-10]. Different conductive particles have been investigated as fillers for these composites such as metallic particles and carbon based particles including carbon fibers, carbon black, carbon nanotubes, reduced graphene oxide, exfoliated graphite and graphene [3-5, 11-16]. In particular, graphene gained increasing interest recently, to be used as an effective filler in polymer composites to enhance their electrical, thermal and mechanical properties, due to its outstanding intrinsic properties, low density and very attractive geometry, i.e., high aspect ratio, high effective surface area and plate-like geometry [10, 12, 17-19]. However, one of the current challenges is the production of cost-effective graphene nanoplatelets at a large industrial scale 
while maintaining a satisfactory level of properties, to allow their use in a broader spectrum of applications, namely: polymer composites applications. In this context, different synthesis methods have been developed during the last decades. So far, the top-down synthesis methods consisting of exfoliation of graphite or graphite derivatives are considered more promising for large-scale production $[10,11]$.

In order to reduce the cost of conductive polymer composites, to maintain the desirable mechanical flexibility/toughness of the polymer matrix and to avoid losing the ease of processing, many research studies focused on the reduction of the percolation threshold concentration, which is extremely sensitive to the dispersion and spatial distribution of the particles. This can be achieved either by using special processing techniques, functionalization of the polymer matrix or the particles, and/or selection of a multiphase polymer matrix as a template for controlled dispersion [11, 12, 20-23]. As far as the two first options are concerned, versatile and successful techniques leading to low and even ultra-low values of percolation threshold have been reported in literature [5, 11, 19, 24-27]. However, the involved processing methods and chemical modifications are often complex and not scalable while the use of industrial techniques is limited. For instance, melt compounding techniques, which are the most attractive processing methods from an industrial point of view, are reported to be relatively unsuccessful in achieving low percolation threshold concentrations [11, 12, 19, 21]. Regarding the option of using multiphase polymer matrices, immiscible polymer blends with co-continuous morphology were found to be a powerful tool to achieve not only the double percolation of the polymer phases and conductive particles, but also the selective localization of the particles in one phase or at the interface; thus reducing considerably the onset of the percolation threshold. This concept was demonstrated for different conductive particles [3, 4, 27-40]. Furthermore, in many cases, the incorporation of particles in the blends and their selective localization often induces changes in the blend's morphology. In particular, their localization at the interface effectively suppresses coarsening and promotes refined and stable blend morphologies [27, 29, 41-45]. In the same context, it was found that plate-like fillers, among them graphene, can adapt better to polymer-polymer interfaces and are subsequently more efficient in suppressing coarsening [42].

In the near-percolation or above percolation regions, the influence of conductive particles networks on electrical and rheological properties of the polymer matrix has been widely investigated. Several scaling relations have been proposed to quantitatively estimate the electrical and rheological percolation threshold concentrations [46, 47]. Besides, in the case of single-phase polymer matrices, the viscoelastic behavior of conductive composites in the molten state has been well-described by a two-phase viscoelastic model that allows the separation of the elastic response of the particles networks from that of the polymer matrix using small amplitude oscillatory shear tests data [48-51]. The main hypothesis of this model is: at high frequencies, the viscous response is dominated by that of the polymer matrix while at low frequencies the elastic response is dominated by that of the networks, formed by particles clusters possibly mixed with a fraction of adsorbed polymer chains. In the case of blends, and especially co-continuous blends, the final arrangement and strength of the electrical and elastic networks depend drastically on the blend's morphology. Simultaneously, the morphology of the blend may evolve in the presence of the particles, especially if they are located at the interface. Such complex interdependence is expected to violate the assumption of the two-phase model and the other scaling relations applied 
in the case of single-phase matrices. Only recently, Filippone et al. [52, 53] applied successfully the two-phase model to PS/PMMA/clay blends featuring drop-in-matrix morphology and even co-continuous morphology. They concluded that relaxation dynamics of the particles elastic network are not affected by the degree of complexity of the matrix microstructure. However, the strength and the space arrangement of the network are sensitive to this factor. Macosko et al. [27] also investigated the modeling of the rheological response of co-continuous PS/PLA blends containing interfacial GN nanoplatelets as a function of the characteristic domain size of the blend. They established a Power scaling law linking the contribution of the interfacial elastic

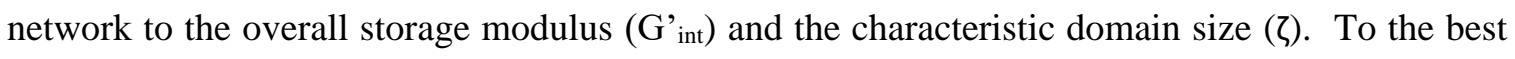
of our knowledge, these models have not yet been used to investigate blends composed of commodity polymers or composites containing mass-produced graphene nanoplatelets.

A strategy to further reduce percolation and at the same time investigate the effect of the conductive network on the morphology of the blends is the use of post-treatments such as thermal annealing. This concept is known as dynamic percolation [30, 54, 55]. In fact, during the processing of conductive polymer nanocomposites, filler networks are established, but they are often in a non-equilibrium thermodynamic state [55-57]. In other terms, the distribution of particles could evolve over time when subjected to an annealing temperature higher than the softening or melting temperature of the matrix. The particles will diffuse and flocculate forming better conductive and elastic networks resulting in a rise of the electrical conductivity and stressbearing ability. In the case of co-continuous blends, the thermal annealing induces, in addition to the rearrangement of particles networks, a more complex mechanism including possible changes in blends morphologies and migration of particles to the interface, which might lower even further the onset of percolation $[28,52]$. In the current literature, only few works have focused on the evolution of the microstructure and the instability of graphene networks in conductive nanocomposites, namely in the case of complex matrix microstructure such as co-continuous blends $[27,28,58]$.

In this work, co-continuous blends of two commodity polymers, linear low density polyethylene (LLDPE) and ethyl vinyl acetate (EVA) containing commercial graphene nanoplatelets synthesized by mechanochemical exfoliation, are studied [59-61]. The blend nanocomposites have been prepared by a facile melt compounding technique. In our previous studies on these materials $[29,62]$, we demonstrated that controlling the processing sequence and in a second step doing a thermal annealing treatment for 2 hours were both effective to tune the localization of GN nanoplatelets in the blend and to lower the percolation threshold concentration. From a practical point of view, the obtained range of electrical conductivity at different working temperatures confirmed that the as-obtained LLDPE/EVA/GN composites can be considered as potential candidates for semi-conductive screen materials and electrostatic dissipation materials.

The present study focuses on the relationships between the viscoelastic response, the electrical conductivity and the co-continuous blend microstructure in LLDPE/EVA/GN materials as well as the evolution of these properties during thermal annealing. Therefore, a combination of morphological, rheological and electrical characterizations was carried out. In particular, the correlation between electrical percolation and rheological percolation was investigated in terms of characteristic percolation concentrations, strength and space arrangement of both elastic and 
electrical networks. A two-phase model was also used to describe the viscoelastic behavior of these materials in an attempt to generalize the mechanisms driving the formation, assembly and stability of interfacial nanoplatelets networks in co-continuous blends to industrially relevant systems consisting of blends of commodity polymers, containing mass-produced plate-like particles, and processed by melt compounding.

\section{Experimental procedure}

\section{II.1. Materials}

Graphene (GN) nanoplatelets, grade Hexo-G V20, were provided by NanoXplore, Inc. This GN grade is produced in large-scale by mechanochemical exfoliation of crystalline graphite. The nanoplatelets surface is functionalized with hydroxyl and carboxyl groups. They have a mean thickness of $20 \mathrm{~nm}$ and average agglomerate flake size of $50 \mu \mathrm{m}$ [29, 60]. Linear low density polyethylene (LLDPE) NOVAPOL PI-2024-A grade of density $0.924 \mathrm{~g} / \mathrm{cm}^{3}$ was purchased from Nova Chemicals and Ethylene vinyl acetate copolymer (EVA) Alcudia PA-540 grade of density $0.937 \mathrm{~g} / \mathrm{cm}^{3}$ was supplied from Repsol. The content of vinyl acetate in EVA resin is around $18 \%$. All the materials were used as received, without further modifications.

\section{II.2. Processing}

The materials were prepared by twin-screw extrusion using a Haake Rheomix OS PTW16. The temperature was fixed to $160^{\circ} \mathrm{C}$ in all zones and the screw speed was adjusted to $100 \mathrm{rpm}$. In a first step, master batches of PE with 25wt\% GN (12.3 vol\%) and EVA with $40 \mathrm{wt} \%$ GN (22.1 vol\%) were prepared. In the rest of the manuscript, the master batches will be referred to as LLDPEMB and EVAMB respectively. In a second step, different EVA/GN and LLDPE/GN composites were prepared by dilution of the masterbatches. In addition, LLDPE/EVA/GN blends were also prepared with the ratio of PE/EVA resins equal to 50wt\%/50wt\%. This composition was selected to achieve a co-continuous morphology, based on the ratio of viscosities measured by capillary rheometer as discussed in our previous manuscript [29]. The viscosity data are also reported in Figure S1 of the electronic supporting information (ESI) file. The blends were prepared from both EVAMB and LLDPEMB master batches and by dilution with proper amounts of LLDPE and EVA. Depending on the master batch used initially, the blend will be referred to as LLDPE/EVA/GN (EVAMB) or LLDPE/EVA/GN (LLDPEMB). The loading of GN in the different materials was varied from 0 to $10 \mathrm{wt} \%$ (4.5 vol\%). Besides, 2 blends with highly unbalanced composition were also processed, in order to help visualizing the localization of GN nanoplatelets. They are identified as LLDPE/mEVA/1wt\% GN and mLLDPE/EVA/1wt\% GN, respectively. In both blends, the loading of GN is equal to $1 \mathrm{wt} \%$ and $\mathbf{m}$ stands for minor phase. More details about these 2 blends are reported in the ESI file.

Before rheological and electrical characterization, the materials were compression molded at $160^{\circ} \mathrm{C}$ and $5 \mathrm{MPa}$ for 5 minutes. In addition, the samples subjected to thermal annealing were kept in the press under $0.8 \mathrm{MPa}$ and $160^{\circ} \mathrm{C}$ for the desired amount of time after the end of the conventional pressing procedure. More details regarding the materials and processing can be found in [29]. In the rest of the manuscript, GN concentrations are expressed in vol\%. 


\section{II.3. Characterization}

The microstructure of the blends at different annealing times has been characterized by means of scanning electron microscopy (SEM). Before imaging, the samples were cryo-fractured in liquid nitrogen and immersed in toluene overnight to dissolve the EVA phase. Subsequently, the obtained cross-sections were gold-sputtered and observed by means of an S3600 Hitachi microscope operated at $5 \mathrm{kV}$ in secondary electrons mode.

The state of dispersion and localization of GN nanoplatelets in the blends depending on the master batch used and the applied thermal annealing was evaluated by transmission electron microscopy (TEM). To obtain 50 to $100 \mathrm{~nm}$ ultra-thin sections, the investigated samples were embedded in epoxy resin and the sectioning was performed using Leica Microsystems UC7/FC7 cryo-ultramicrotome operated at $-160^{\circ} \mathrm{C}$. The sections were then transferred onto formvar coated 200-mesh Cu TEM grids, which were washed carefully with distilled water in order to remove sugar solution and then dried. Imaging was carried out with a FEI Tecnai G2-F20 Scanning Transmission Electron Microscope operated at an accelerating voltage of $200 \mathrm{kV}$.

The shear viscosities of unfilled LLDPE and EVA resins, LLDPEMB and EVAMB master batches and selected LLDPE/EVA/GN composites were measured at $160^{\circ} \mathrm{C}$ and different shear rates ranging from 10 to $1000 \mathrm{~s}^{-1}$, using an Instron SR20 capillary rheometer equipped with a capillary of $40 \mathrm{~mm}$ in length and $1 \mathrm{~mm}$ in diameter. The measurements were rectified using Rabinowitch correction.

The dynamic viscoelastic behavior of the materials was characterized through different rheological measurements performed by an Anton-Paar MCR501 rotational rheometer operated in a parallel-plate configuration, at $160^{\circ} \mathrm{C}$ and under a nitrogen atmosphere. Discs of $25-\mathrm{mm}$ diameter and 1-mm thickness were used to perform the measurements. At first, dynamic strain sweep tests (DSST) were performed at 3 different angular frequencies, 1, 10 and $100 \mathrm{rad} . \mathrm{s}^{-1}$, in order to determine the region of linear viscoelasticity (LVE). Subsequently, small amplitude oscillatory shear (SAOS) tests and time sweep tests were performed at amplitude strains selected in the LVE region. SAOS tests were performed in the angular frequency range from $10^{-2}$ to 300 rad. $\mathrm{s}^{-1}$ while time sweep tests were performed for 70 minutes for materials prepared from EVAMB and 2 hours for materials prepared from LLDPEMB, at a fixed angular frequency equal to $10^{-1} \mathrm{rad} . \mathrm{s}^{-1}$.

The complex dielectric permittivity was evaluated using a Novocontrol broadband dielectric spectrometer in the frequency range from $10^{-2}$ to $300 \mathrm{~Hz}$ under an excitation voltage of $3 \mathrm{~V}_{\text {RMS }}$. To perform the measurements, specimens of $40 \mathrm{~mm}$ in diameter and around $0.4 \mathrm{~mm}$ in thickness were placed between two parallel brass plated electrodes after being coated with $10 \mathrm{~nm}$ layer of platinum. The complex conductivity was then derived from the complex dielectric permittivity. The conductivity values presented in this manuscript correspond to the real part of the complex conductivity measured at $10^{-2} \mathrm{~Hz}$. 


\section{Results and discussion}

\section{III.1. Composites processability and morphology of the blends}

The results of shear viscosity as function of shear rate, reported in Figure S1 of the electronic information supporting (ESI) file, show that in the region of shear rates around $100 \mathrm{~s}^{-1}$, which is the range of shear rates expected during extrusion [63], the viscosities of LLDPE, EVA, LLDPE/4.5GN, EVA/4.5GN and LLDPEMB (12.3 vol\% GN) are similar, i.e. no increase in melt viscosity. EVA/4.5GN shows even slightly lower viscosity than that of pure EVA, which is most likely attributed to a lubricant effect of GN nanoplatelets. In fact, the 2D geometry of the platelets helps them sliding over one another, leading to low resistance to shear flow [64]. Only the viscosity of EVAMB ( $22.1 \mathrm{vol} \% \mathrm{GN})$ is few times higher but still not expected to deteriorate the processability of the composites.

In terms of morphology, since the viscosities of LLDPE and EVA resins and their volume fractions in the blends are similar, a co-continuous morphology is expected for a 50/50 blend concentration [65]. SEM images reported in our previous publications $[29,61]$ confirm the achievement of co-continuous morphology in the case of the unfilled LLDPE/EVA blend as well as in the case of LLDPE/EVA/GN composites prepared from both master batches. Furthermore, it was observed that LLDPE/EVA/GN composites feature a refinement of their morphologies with increasing loading of GN. This reduction of domain sizes is most likely attributed to suppression of coarsening and alteration of interfacial rheology by the presence GN nanoplatelets accumulated at the interface. In fact, the platelets may act as physical barriers preventing the direct contact between the polymer domains. Besides, the interfacial particles could alter the interface and retard the relaxation phenomena involved in the coarsening process [27, 28, 52]. A higher degree of morphology refinement was observed in blend composites prepared from EVAMB, as reported in [29]. The observed increase of EVAMB viscosity might favor the breakup mechanism [42] and be subsequently responsible of this behavior.

\section{III.2. Localization of GN nanoplatelets in LLDPE/EVA blend}

The localization of particles inside an immiscible blend depends primarily on the thermodynamics of the system [36, 44]. Therefore, depending on the affinity of GN particles with each phase, their localization can be tuned. A prediction of GN localization in LLDPE/EVA blend based on wetting theory was elaborated in our previous work and revealed that GN nanoplatelets might have slightly higher affinity with EVA phase due to its inherent polar groups. More details can be found in reference [29]. Because of this slight affinity, GN nanoplatelets tend to selectively locate in EVA phase or to stay at the interface. The kinetics of the processing constitutes another important factor that affects the localization of particles [28, 66]. For instance, in order to help tailoring the localization of GN nanoplatelets in LLDPE/EVA co-continuous blend, two processing sequences were used to prepare LLDPE/EVA/GN materials, depending on the master batch selected, as mentioned previously in the processing section and reported in [29]. More specifically, GN nanoplatelets were either premixed with EVA, and then mixed with LLDPE (in this case EVAMB is used), or premixed with LLDPE, and then mixed with EVA. In particular, if GN nanoplatelets are premixed with the thermodynamically less-favorable LLDPE phase, they tend to migrate during the processing to the EVA phase. Depending on the 
compounding time and the matrix viscosity at the processing shear rate, they will eventually reach the EVA phase or stay confined at the interface, which constitutes an energy trap, as reported in some recent studies regarding particles filled immiscible blends [27, 28]. In addition to thermodynamics and kinetics, the geometry of the particles and the morphology of the blend were also found to affect the final localization of particles. In particular, the thinnest particles will be the fastest to transfer from one phase to another [67].

In the case of the studied LLDPE/EVA/GN materials, SEM images reported in [29] confirmed indeed the presence of more interfacial GN platelets gathered at the interface in the case of LLDPE/EVA/GN (LLDPEMB). In order to confirm further the occurrence of this selective localization, TEM was also performed on LLDPE/EVA/1.3GN composites prepared from both LLDPEMB and EVAMB. In Figure 1(a) and 1(b), the images corresponding to LLDPE/EVA/1.3GN (EVAMB) composite are reported, while in Figure 1(c) and 1(d), the images corresponding to LLDPE/EVA/1.3GN (LLDPEMB) composite are presented. At a first glance, the images taken for both materials look similar. We believe that the reason for this is the relatively weak electronic contrast between LLDPE and EVA polymers, which actually becomes worse in the presence of GN. However, it seems that, in both sets, GN nanoplatelets are mostly located in only one phase in each case which is most likely the master batch phase, i.e. EVA phase in the case of LLDPE/EVA/1.3GN (EVAMB) and LLDPE in case of LLDPE/EVA/1.3GN (LLDPEMB). To visualize better this selective localization of GN, the TEM images of the 2 blends with highly unbalanced compositions, respectively mLLDPE/EVA/1wt\% GN and LLDPE/mEVA/1wt\% GN, are presented in section 2 of the ESI file.

In fact, during the processing time, a complete diffusion of GN nanoplatelets to EVA phase or their stabilization at the interface are not expected to be reached. This hypothesis will be validated later when discussing the thermal annealing results. In the case of LLDPE/EVA/1.3GN (LLDPEMB) composite, a more obvious fraction of GN nanoplatelets can be observed at the interface, as indicated by the arrows in Figure 1(d). This observation supports the idea that GN migration is encouraged when the particles are premixed with the thermodynamically lessfavorable LLDPE phase.

Figure 1: TEM of (a) (scale bar: $1 \mu \mathrm{m}$ ) and (b) (scale bar: $200 \mathrm{~nm}$ ) LLDPE/EVA/1.3GN (EVAMB), (c) (scale bar: $500 \mathrm{~nm}$ ) and (d) (scale bar: $200 \mathrm{~nm}$ ) LLDPE/EVA/1.3GN (LLDPEMB)

\section{3. Formation of GN networks in LLDPE/EVA co-continuous blends compared to LLDPE and EVA single-phase polymers}

Dynamic strain sweep tests were performed on the different composites sets, LLDPE/GN, EVA/GN and LLDPE/EVA/GN, to evaluate their LVE region and its dependence on GN loading. The results are reported in Figure 2. In particular, in the LVE region, the dynamic moduli, namely the storage modulus G', are expected to exhibit a plateau up to a certain critical strain $\left(\gamma_{c}\right)$. Above this strain, the viscoelastic moduli drop. In the presence of fillers, it was already observed and 
reported in the literature that this drop occurs at a $\gamma_{c}$ value below that of the unfilled polymer matrix. This reduction of the LVE region is attributed to the disruption of filler networks at increasing strain values. This effect is known as Payne effect and is commonly used as a tool to estimate the rheological percolation threshold [68]. From Figure 2(a), it can be deduced that the rheological percolation threshold is expected between 7 and $9.6 \mathrm{vol} \% \mathrm{GN}$ in the case of EVA/GN composites and between 2.2 and $4.5 \mathrm{vol} \%$ in the case of LLDPE/GN composites, respectively. From Figure 2(c), the rheological percolation is potentially expected between 0.4 and $1.3 \mathrm{vol} \%$ GN in the case of LLDPE/EVA/GN (LLDPEMB) and well below $1.3 \mathrm{vol} \% \mathrm{GN}$ in the case of LLDPE/EVA/GN (EVAMB). Moreover, comparing the observed Payne effect in both LLDPE/EVA/1.3GN (LLDPEMB) and LLDPE/EVA/1.3 (EVAMB), one can conclude that the rheological percolation is expected at lower GN concentrations in the case of EVAMB based materials. Besides, it is worth noting that both LLDPE/EVA/GN (LLDPEMB) and LLDPE/EVA/GN (EVAMB) exhibit almost identical rheological behavior starting at GN loadings equal to $2.2 \mathrm{vol} \%$ and higher, which is in line with their similar morphologies observed by SEM, as reported in our previous study [29]. Hence, it can be concluded from the DSST tests that the onset of the rheological percolation threshold has been considerably reduced in LLDPE/EVA/GN blends compared to LLDPE/GN and EVA/GN single-phase matrix composites. These first estimations of the percolation threshold concentrations obtained from the DSST tests will be correlated with the results of SAOS tests, time sweep tests as well as the electrical conductivity measurements discussed in the next sections.

Figure 2: DSST results (G' vs. strain plots) of selected (a) EVA/GN and LLDPE/GN and (b) LLDPE/EVA/GN composites (performed at 1 rad.s $\mathrm{s}^{-1}$ and $160^{\circ} \mathrm{C}$ ), (c) inset from (b) showing selected materials

\section{III.4. Time and temperature dependence of rheological and electrical properties in correlation with blend's morphologies}

It has been repeatedly reported that electrically conductive polymers are thermodynamically instable systems. This means that above the melting or the softening temperature of the host polymer matrix, the clusters of the conductive particles tend to reassemble into bigger structures because of the inter-particle attraction. This flocculation process will be achieved through the diffusion of the particles and even their migration from one phase to another, leading in some cases to a reduction in the onset of the percolation threshold as reported by several studies [27-29, $49,52,54-58]$.

The kinetics of this process, known as the dynamic percolation, can be monitored by looking at the evolution of the linear viscoelastic moduli as function of thermal annealing time, at a temperature higher than the melting or softening point and a frequency $\omega$ low enough to consider negligible both the elastic contribution of the matrix $[51,53]$ and the effect of shear. These time sweep tests can be considered as a thermal annealing experiment. For this study, the time sweep tests were performed on selected LLDPE/GN, EVA/GN and LLDPE/EVA/GN materials at 0.1 rad. $\mathrm{s}^{-1}$ and at $160^{\circ} \mathrm{C}$ for 2 hours. The time-dependent evolutions of the storage modulus $\mathrm{G}^{\prime}$ and the loss modulus G' "are reported in Figure 3. In particular, for each material, G' and G' were 
normalized over their initial values $\mathrm{G}_{0}$ and $\mathrm{G}$ " ${ }_{0}$, which are the values of $\mathrm{G}$ ' and $\mathrm{G}$ " at the beginning of the test, respectively. The evolution of electrical conductivity was investigated as function of annealing time up to 2 hours as well, to evaluate the potential changes induced by the rearrangement of GN networks on the values of electrical conductivity and the onset of electrical percolation threshold and eventually correlate the effects of thermal annealing on rheological percolation and electrical percolation. The results are reported in Figure 4. The evolution of the composites morphologies as function of annealing time was investigated as well through SEM images taken at selected annealing times. The micrographs are reported in Figure 5. The characteristic domain size of each blend composite was estimated from the SEM images using equation (1) described below [27]. At least two different images at a magnification of 300x were considered for each material. The results are reported in Figure 6. However, due to the complexity of the tridimensional morphology of co-continuous blends, it is worth noting that this 2D method might induce some imprecision in the estimation of the interfacial area and consequently the characteristic domain size. Some studies have used confocal microscopy to obtain more precise structural characterization [44].

$$
\zeta=\frac{A_{S E M}}{L_{\text {int }}}
$$

Where:

A AEM: total area of the SEM image.

$\mathrm{L}_{\text {int: }}$ interface length estimated by image $\mathrm{J}$

The plot of G' and G' vs. annealing time corresponding to LLDPE/EVA/GN materials, reported in Figure 3(a) and Figure 3(b) respectively, show that G' and G" of the unfilled LLDPE/EVA blend and that of LLDPE/EVA/0.4GN (LLDPEMB) composite remain almost constant up to 2 hours of annealing. The quasi-unchanged $G^{\prime}$ in these materials indicates the absence of any important change of morphology during this annealing time at $160^{\circ} \mathrm{C}$. In particular, in the absence of GN or in the presence of only small GN amount, the coarsening is expected to occur and be completed very fast, partially during processing, compression molding and the temperature stabilization step before the start of rheological tests. The remaining LLDPE/EVA/GN composites share the same qualitative behavior with an initial increase of their G' during the early stage of annealing: up to 25-30 min, followed by more steady state behavior over time. A similar trend is noticed for the loss modulus G", but the increase is considerably less pronounced (Figure 3(b)). At this level, these particular changes in viscoelastic moduli can be attributed to several factors including considerable alteration of GN nanoplatelets spatial arrangement, potential changes of the blend morphologies and eventually thermal degradation. Some of these factors will be discarded in the upcoming discussions.

From Figure 3(c) and Figure 3(d), showing respectively G' vs. annealing time and G" vs. annealing time plots corresponding to LLDPE/GN and EVA/GN single-phase composites, it can be seen that the pure resins feature constant moduli as function of annealing time while the composites exhibit progressive increase of their G' and G" with increasing annealing time. This increase is more important with increasing loading of GN and also more obvious in the case of G' (compared to G'). Unlike the case of multi-phase LLDPE/EVA/GN composites, the increase of 
G' of LLDPE/GN and EVA/GN is observed at remarkably higher GN loadings, is more progressive over time and does not seem to reach a steady state up to 2 hours of thermal annealing. This behavior might indicate the formation of more stable networks in the case of LLDPE/EVA/GN materials. In particular, interfacial GN nanoplatelets are most-likely responsible for this stability as they require more energy to be detached from the interface [27].

The growth of the moduli at rest, essentially the elasticity, is a characteristic rheological behavior of nano-filled polymers, especially in the case of plate-like fillers. It has been reported that the growth of G' at low fractions of fillers reflects an increase of their effective volume fraction, after losing the initial alignment imposed by the compression applied when loading the sample [53, 69]. At higher filler loadings, the growth of elasticity is more important due to flocculation/rearrangement of particles causing polymer confinement. Above the percolation threshold, an elastic filler network is established, increasing further the overall elasticity. Ultimately, once the steady state is reached, the system can be seen as a tridimensional network of nanoparticles clusters distributed within the polymer matrix, and the viscoelastic response is a combination of the responses of the two components. As observed, the response of the network is essentially elastic (more important growth of $\mathrm{G}^{\prime}$ ). A viscous contribution might be present as well and is attributed to slow relaxations dynamics due to rearrangement/redistribution of clusters. However, this contribution is minor [49]. Within the context of this work, the viscous part of the network response will be neglected. Also, the elastic part of the matrix response will not be considered since the time sweep tests are performed at low frequencies. Hence, in these systems, the elastic response is totally related to the fillers network while the viscous response is entirely attributed to the polymer matrix. This assumption has been considered in several studies performed on conductive polymer nanocomposites $[49,52,53]$. Its validity in the context of this work will be discussed in a later section.

The diffusivity of plate-like particles in a certain medium can be determined using equation (2) describing the rotary diffusivity of rigid disks $\left(\mathrm{D}_{\mathrm{r} 0}\right)$ [53]. If we consider a shear rate of $100 \mathrm{~s}^{-1}$, the viscosities of EVA and LLDPE are almost equal according to Figure S1 from the ESI file (490 Pa.s for EVA vs. 460 Pa.s for LLDPE) and consequently the diffusivity of GN particles in both materials is expected to be similar. At shear rates lower than $100 \mathrm{~s}^{-1}$, LLDPE exhibits slightly lower viscosity values.

The characteristic time scale for structural rearrangements can be roughly estimated as the inverse of the rotary diffusivity [53]. Simultaneously, this quantity can be deduced experimentally from the results of time sweep tests showing a progressive increase of the storage modulus G' during the first 28 minutes of annealing, which is believed to be mainly caused by the flocculation/diffusion of particles and redistribution of GN networks as well. Using equation (2), the average lateral size of particles that are able to diffuse according to the value of diffusion time scale deduced from time sweep tests is around $250 \mathrm{~nm}$. Particles with larger lateral dimensions will require higher temperature/lower viscosity to be able to diffuse. This point will be discussed further later in the manuscript in the analysis of TEM data. It is worth noting that equation (2) can be only applied for GN platelets that are either in LLDPE phase or EVA phase. The rearrangements of particles that are already confined at the interface might involve more energetic processes that are not considered in equation (2) such as the detachment of particles 
from the interface [53]. Also, in the case of particles diffusing toward a polymer interface, the interfacial energy between GN and each polymer may also affect the rate of diffusion.

$$
D_{r 0}=\frac{3 k_{B} T}{4 \eta d^{3}}
$$

Where:

$\mathrm{T}$ : temperature

$\mathrm{k}_{\mathrm{B}}$ : Boltzmann constant

$\eta$ : is the viscosity of the suspending medium

$d$ : is the disk diameter.

Figure 3: Time sweep tests of selected LLDPE/GN, EVA/GN and LLDPE/EVA/GN composites at $10^{-1} \mathrm{rad}_{\mathrm{s}} \mathrm{s}^{-1}$ and $160^{\circ} \mathrm{C}$ : (a) $\mathrm{G}^{\prime} / \mathrm{G}^{\prime}{ }_{0}$ vs. annealing time of LLDPE/EVA/GN multi-phase composites, (b) G' $/ \mathrm{G}$ ' ${ }_{0}$ vs. annealing time of LLDPE/EVA/GN multi-phase composites, (c) G'/G' ${ }_{0}$ vs. annealing time of LLDPE/GN and EVA/GN single-phase composites and (d) G'/G"' vs. annealing time of LLDPE/GN and EVA/GN single-phase composites

To understand better the phenomena that occur during the early stage of annealing, comparison with electrical and morphological behaviors is necessary. Plots of electrical conductivity vs. annealing time reported in Figure 4 show that the conductivity of the unfilled LLDPE/EVA blend didn't change with annealing time. However, for all LLDPE/EVA/GN multi-phase composites a remarkable increase of conductivity occurs also at the first 25-28 minutes of thermal annealing, which indicates further improvement of GN networks due to particles diffusion and flocculation and their potential migration to the interface. Then, the electrical conductivity tends to stabilize. Overall, this behavior is consistent with the results of time sweep tests discussed earlier. In the case of LLDPE/2.2GN and EVA/4.5GN single-phase composites, a progressive increase of the electrical conductivity is observed as function of annealing time up to 100 minutes. However, stability is not reached. This behavior is also in agreement with the time sweep tests.

It is worth noting that in this study the rheological measurements were done on molten samples while the electrical conductivity measurements were performed on solid samples, in which the thermal history and crystallization could have altered the conductive networks. However, overall, the good agreement between rheological and electrical behaviors suggests that crystallization did not alter considerably the filler network in the studied system. It also supports the idea that the diffusion and rearrangement of GN nanoplatelets is at the origin of the improvement of elastic and conductive networks. In addition, the accumulation of interfacial nanoplatelets in LLDPE/EVA/GN multi-phase composites promotes the stability of the networks formed upon annealing.

Figure 4: Electrical conductivity as function of thermal annealing time at $160^{\circ} \mathrm{C}$ of selected EVA/GN, LLDPE/GN and LLDPE/EVA/GN composites

SEM images of annealed LLDPE/EVA/GN composites reported in Figure 5 and the plots of characteristic domain size vs. annealing time, reported in Figure 6 show that important coarsening (increase of the characteristic domain size द) occurs mainly during the first 30 minutes of 
annealing of LLLDPE/EVA/1.3GN and LLDPE/EVA/2.2GN composites (from both master batches). Then, the morphologies tend to be more stable over time. This stability of morphology correlates well with the observed electrical and rheological behaviors. In the case of the composites prepared from EVAMB, the relatively high viscosity of the master batch might be the reason of their finer morphologies compared to materials prepared from LLDPEMB.

The observed coarsening during thermal annealing is equivalent to a reduction of the interface area. Therefore, the increase of rheological and electrical quantities during the first 30 minutes of annealing is most likely due to a simultaneous diffusion of GN to the interface and important coarsening (due to coarsening, less GN is required to saturate the interface). Then, the coarsening is expected to proceed until the strength of the interfacial structure formed by the nanoplatelets overcomes the interfacial tension. As a consequence, the morphology of the blend is stabilized over time by the presence of interfacial GN platelets. It is worth noting that this rearrangement of GN networks is occurring at the same timescale independently from the master batch used, considering time sweep tests and electrical conductivity tests. Hence, it seems that during annealing GN is diffusing and rearranging from both polymer phases, following the same trend, which infers that GN platelets may have no preferential phase and tend to migrate to the interface during thermal annealing whether they are premixed with LLDPE or EVA.

Figure 5: Evolution of morphology of selected LLDPE/EVA/GN composites at different annealing times and at $160^{\circ} \mathrm{C}$ : (a1) to (a4) SEM images of LLDPE/EVA/1.3GN (EVAMB) at respectively $0,28,75$ and 95 mins of annealing, (b1) to (b4) SEM images of

LLDPE/EVA/1.3GN (LLDPEMB) at respectively 0, 28, 75 and 95 mins of annealing, (c1) to (c4)

SEM images of LLDPE/EVA/2.2GN (EVAMB) at respectively $0,25,70$ and 100 mins of annealing, (d1) to (d4) SEM images of LLDPE/EVA/2.2GN (LLDPEMB) at respectively 0, 25, 70 and 100 mins of annealing (Scale bar $=100 \mu \mathrm{m}$ for all images)

Figure 6: Characteristic domain size $\zeta$ vs. annealing time at $160{ }^{\circ} \mathrm{C}$ of selected LLDPE/EVA/GN composites

To validate the hypothesis of diffusion and migration of GN platelets to the interface during annealing, LLDPE/EVA/1.3GN (LLDPEMB) composite was also observed by TEM at 28 mins of annealing, as shown in Figure 7(b-d). Figure 1(c) illustrating LLDPE/EVA/1.3GN (LLDPEMB) before annealing is presented again in Figure 7(a) to compare the morphologies before and after thermal annealing. It can be seen that before annealing, GN, which is believed to be mostly located in LLDPE phase in this configuration, is tending to cross the interface, as seen in Figure 7(a). However, after 28 mins of annealing (where the highest changes of G' and electrical conductivity were observed), a clear migration and organization of GN at the interface and close to it is observed (Figure 7(b-d)). Furthermore, the interface seems to be well defined and covered by thin and flexible GN nanoplatelets while before annealing, GN platelets didn't follow the contour of the interface. It is worth noting that at this composition, the interface is still not saturated by GN nanoplatelets. Two sets of nanoplatelets can be distinguished: 
- Very thin nanoplatelets, having only few layers and a lateral size around $1 \mu \mathrm{m}$ or less (indicated by the yellow dashed arrows in Figure 7(b) and Figure 7(c)), that are at the interface or close to it. This set is predominant.

- Few thick nanoplatelets settled in parallel of each other and following well the boundary.

This space arrangement of nanoplatelets is in agreement with the scheme described by Filippone et al. [52]. Compared to the estimation of lateral sizes obtained by equation (2), the TEM images reveal that nanoplatelets larger than $250 \mathrm{~nm}$ are also able to diffuse. This fact supports that the diffusion of particles does not only depend on the viscosity of the surrounding medium but may also depend on the chemical affinity between the species.

Overall, we can conclude that the observed increase of G' and electrical conductivity upon annealing is indeed due to the migration of more GN nanoplatelets to the interface which counterbalances the effect of the simultaneous coarsening of the blend morphology. This interface can actually be used as a template on which the plate-like particles can lie, offering the possibility of well-tailored tridimensional structure [27, 52].

Figure 7: TEM images of LLDPE/EVA/1.3GN (LLDPE MB) (a) before annealing at $160^{\circ} \mathrm{C}$ (scale bar $=500 \mathrm{~nm}$ ) and (b-d) after annealing for $28 \mathrm{~min}$ at $160^{\circ} \mathrm{C}$ : (b) scale bar $=1 \mu \mathrm{m}$, (c) scale bar $=1 \mu \mathrm{m}$ and (d) scale bar $=500 \mathrm{~nm}$

\section{5. Modeling the rheological response using the Two-Phase model}

As shown by the time sweep tests and microscopy, the main evolution of morphology and viscoelastic moduli occurs during the first 30 minutes of thermal annealing. Therefore SAOS tests were performed on different LLDPE/EVA/GN composites after oscillatory shear flow at 0.1 $\operatorname{rad} . \mathrm{s}^{-1}$ and $160^{\circ} \mathrm{C}$ for 30 minutes, in order to stabilize their morphologies. After this stabilization step, the interfacial network of nanoparticles and the blend morphology are expected to be fully evolved. These data could then be used to characterize the morphology of the blend and evaluate the effect of the presence of graphene. G' vs. angular frequency $\omega$ and G" vs. $\omega$ plots related to LLDPE/EVA/GN (LLDPEMB) are presented in Figure 9(a) and Figure 9(b), respectively and those related to LLDPE/EVA/GN (EVAMB) are presented in Figure 9(c) and Figure 9(d), respectively. Compared to SAOS measurements performed without the 30 mins annealing step, it was found that G' of the composites exhibit more well-established plateaus at low frequencies (SAOS curves without the stabilization step are not presented in this manuscript).

It is worth noting that unfilled LLDPE/EVA blend exhibits a non-terminal behavior compared to pure LLDPE and EVA resins as reported in our previous work [29], reflected by a decrease in the slope of G' vs. $\omega$ plot at low frequencies. Besides, all LLDPE/EVA/GN multi-phase composites with GN concentrations equal to or higher than 1.3 vol\% feature a clear increase of their moduli at low frequencies. This increase is more obvious in the case of the elastic modulus G'. Furthermore, a zero-slope of G' vs. $\omega$ plot is observed and extended to higher frequencies with increasing loading of GN. This non-terminal behavior was observed in many nanocomposites 
based on single-phase polymer matrix and has been attributed to the achievement of the percolation threshold and the formation of GN networks confining the polymer chains and arresting their relaxation dynamics. The behavior of such nanocomposite is dominated by the elastic particles network at low frequencies and has been modeled using a two-phase model [4853]. This model describes the complex viscoelastic behavior of the nanocomposites above the percolation threshold as the independent contributions of two dynamical components: the predominantly viscous polymer matrix and the mainly elastic particles network. The validity of this model in describing a particular polymer nanocomposites system is verified through the successful building of a master curve of all the samples above the percolation threshold [48, 52] . The importance of building of a master curve, in addition to revealing the elastic response mechanism in the composites, resides also in allowing the estimation of the elasticity contribution of weak filler networks; i.e. samples above percolation but not showing necessarily a low frequency plateau modulus [52].

Figure 8: SAOS tests performed at $160^{\circ} \mathrm{C}$ after stabilization for 30 mins on: LLDPE/EVA/GN (LLDPEMB) composites (a) G' vs. $\omega$ and (b) G' 'vs. $\omega$ and LLDPE/EVA/GN (EVAMB) composites (c) G' vs. $\omega$ and (d) G', vs. $\omega$

The procedure to build the master curve and the physical meaning of the scaling parameters are described in details elsewhere $[48,52]$. Within the context of this study, we will recall only the main points. Mainly, three factors have to be determined for each nanocomposite. First of all, an amplification factor $\mathrm{B}(\Phi)$ which accounts for the hydrodynamic effects and can be determined as the ratio of the complex shear moduli of the filled composite $G^{*}(\omega)$ and the neat matrix $G^{*}{ }_{m}(\omega)$ at a frequency $\omega$ high enough to consider the nanocomposite response governed by the polymer matrix: $\mathrm{B}(\Phi)=\mathrm{G}^{*}{ }_{c}(\omega) / \mathrm{G}^{*}{ }_{\mathrm{m}}(\omega)$. In addition, a vertical shift factor $\mathrm{b}_{\Phi}$ is considered, which represents the $\varphi$-dependent network elasticity. $b_{\Phi}$ is set as the low frequency plateau modulus as a rough estimate, which is often clear for the nanocomposites well above percolation. Finally, a horizontal shift factor $\mathrm{a}_{\Phi}$ is also considered, which represents the frequency separating the regime in which the behavior is dominated by the particle network from that in which the polymer governs the response. It is determined as the frequency at which the viscous modulus of the neat polymer, properly amplified by $\mathrm{B}(\Phi)$, crosses the network elasticity estimated by $\mathrm{b}_{\Phi}$.

Recently, it was claimed by Filippone et al. that the two-phase model can be extended to the case of polymer blends with drop-in-matrix morphology and even co-continuous morphology [52, 53], whose intrinsic elasticity could also contribute to the overall elasticity and subsequently mask that of the nanoparticles network at low particles loadings [52]. In particular, the authors demonstrated that it is possible to represent PS/clay single-phase composites and PS/PMMA/clay multi-phase composites using the same master curve. In the present study, it was possible to build a master curve of all LLDPE/EVA/GN nanocomposites with GN loading higher than $1.3 \mathrm{vol} \%$, as shown in Figure 9. Besides, it was found that the scaled curves of LLDPE/GN and EVA/GN composites above the percolation threshold are also superimposed on the same master curve. The two shift factors, $a_{\Phi}$ and $b_{\Phi}$, as well as the amplification factor $\mathrm{B}(\Phi)$ corresponding to each nanocomposite are reported in Table S1 of the ESI file. 
Overall, the quality of the scaling and the overlay of the different curves support the common nature of the elastic behavior in all these composites, independently from the polymer matrix. G' curve of LLDPE/EVA/0.4GN composite was not scalable which implies that the percolation threshold falls between $0.4 \%$ and $1.3 \mathrm{vol} \%$, considering the hypotheses of the model. Looking more in depth, the overlay of all scalable curves is good up to a $\omega / a_{\Phi}$ ratio equal to 100 . At higher values, few G' curves such as, LLDPE/4.5GN and EVA/9.6GN, are slightly shifted from the built master curve. This is probably due to the dominance of LLDPE and EVA resins responses in this region of frequencies, which may differ from that of the LLDPE/EVA blend.

\section{6. Rheological and electrical percolation thresholds}

The successful modeling of the viscoelastic behavior of LLDPE/EVA/GN materials with cocontinuous morphology using the two-phase model indicates that the viscoelasticity of the filled blend can be described irrespective of the complexity of its morphology [52, 53]. Therefore, it is legitimate to use approaches initially proposed for systems with single-phase matrix such as the scaling Power law predicting that the elasticity of the network $\mathrm{G}_{0}^{\prime}$ to grow with particles volume fraction $\Phi$ following equation (3) [52, 58]:

$$
G_{0}^{\prime}=A\left(\Phi-\Phi_{c}\right)^{t}
$$

Where

- $A$ : scaling factor reflecting the strength of the network

- $\Phi_{c}$ : percolation threshold concentration

- $t$ : critical exponent depending on the aspect ratio of the filler and describing the spatial distribution of the network

In addition to the prediction of $\Phi_{c}$, the analysis of the critical exponent gives an insight about the network structure $[49,52,58]$, which in this case might depend on the complexity of the morphology of the polymer matrix.

Figure 9: Master curve showing the layover of different LLDPE/GN, EVA/GN and LLDPE/EVA/GN materials

In Figure 10, the vertical shift factors $b_{\Phi}$, which were used to built the master curve and represent the network elasticity, are plotted as function of reduced filler content $\Phi-\Phi_{c}$. The data fitted to a Power law, described in Equation (3), gives a good estimate of $\Phi_{\mathrm{c}}$ as the value corresponding to the highest regression coefficient. The fitting parameters are also reported in Figure 10. The estimated values of $\Phi_{\mathrm{c}}$ are $1 \mathrm{vol} \%(2.4 \mathrm{wt} \%)$ and $0.35 \mathrm{vol} \%(0.8 \mathrm{wt} \%)$ for LLDPE/EVA/GN (LLDPEMB) and LLDPE/EVA/GN (EVAMB) sets, respectively, which means that LLDPE/EVA/GN (EVAMB) system achieves the rheological percolation threshold at lower GN concentrations. This is in agreement with the rheological percolation threshold values predicted in section III.3 by Payne effect. On the other hand, the value of the critical exponent $t$ is slightly higher in the case of LLDPE/EVA/GN (LLDPEMB) compared to LLDPE/EVA/GN (EVAMB): 
2.33 vs. 2 . Also, the strength of the network, reflected by the scaling factor $A$, is higher in the case of LLDPE/EVA/GN (LLDPEMB) materials $\left(4 \times 10^{+06} \mathrm{~Pa}\right.$ vs. $\left.6.67 \times 10^{+05} \mathrm{~Pa}\right)$, probably due to the existence of a higher fraction of nanoplatelets located at the interface in this set.

Compared to literature, the estimated rheological percolation threshold concentrations are higher than those reported recently by different groups such as Gao. et al [58] for SAN/chemically reduced graphene oxide nanocomposites prepared by coagulation method $(0.1 \mathrm{vol} \%)$ or ABS/Graphene prepared by chemical blending ( $<0.24$ vol\%) [68] and Macosko et al. [24] for polyurethane acrylate/thermally reduced graphene nanocomposites prepared by in situ polymerization $(0.5 \mathrm{wt} \%)$. However, they remain close to the range of percolation threshold limit, between 0.5 and $1 \mathrm{wt} \%$, reported recently for polyolefin/graphene nanocomposites [19]. Besides, they are lower than the rheological percolation values reported in other studies such as Filippone et al. $[49,50]$ for PS/GN nanoplatelets nanocomposites prepared by chemical blending (1.8 vol\%).

In terms of the critical exponent $t$, the values estimated in this study remain within the range reported in literature, especially for nanocomposites based on multiphase polymer matrices and filled with plate-like particles $[49,50,52,53]$. In the context of rheological percolation, this exponent is indicative of the mechanism through which the particles resist stress. In particular, it has been reported that values of $t$ lower than 2.1 correspond to particle networks mediated by the polymer matrix chains while values of $t$ higher than 3.75 correspond to networks established directly between strong interacting particles [50]. Hence, the networks established in the studied LLDPE/EVA/GN materials (both sets) follow rather the first case. Moreover, in the case of multiphase polymer matrices, lower critical exponents were reported compared to single-phase matrices. In fact, in this case the space arrangement of particle networks is driven by the polymerpolymer interface which leads to lower sensitivity to the increasing filler content above percolation [52].

Figure 10: Network elasticity as a function of reduced filler content: $b_{\Phi}$ vs. $\left(\Phi-\Phi_{c}\right)$ plots related to LLDPE/EVA/GN composites (solid squares) and power-law fitting to the experimental data (solid lines)

In order to evaluate the correlation between electrical and rheological percolation, the electrical percolation threshold was also estimated using an equation formally identical to equation (3) with $\sigma_{0}$ instead of $\mathrm{G}_{0}{ }_{0}$. The best fittings corresponding to both sets of composites are reported in Figure 11 , with $\Phi_{\mathrm{c}}$ values also selected to correspond to the highest regression coefficient. In particular, the experimental conductivity values before annealing for 2 hours (Figure 11(a)) and after annealing for 2 hours (Figure 11(b)) were considered for the analysis, to emphasize the role of annealing on the electrical percolation threshold. Before annealing, $\Phi_{c}$ values were found equal to $1.7 \mathrm{vol} \%$ and $2.5 \mathrm{vol} \%$ for LLDPE/EVA/GN (LLDPEMB) and LLDPE/EVA/GN (EVAMB) sets, respectively. However, after annealing, $\Phi_{\mathrm{c}}$ values were found equal to $1.21 \mathrm{vol} \%(2.8 \mathrm{wt} \%)$ and $0.5 \mathrm{vol} \%$ (1.2 wt\%)for LLDPE/EVA/GN (LLDPEMB) and LLDPE/EVA/GN (EVAMB) sets, respectively. These values are close to those estimated for rheological percolation as discussed above. As a consequence, these findings support the strength of rheological characterization as a tool to study dynamic percolation threshold in conductive polymer composites and to predict the 
percolation threshold with accuracy. Also, this agreement between $\Phi_{c}$ values estimated from rheological and electrical data suggests that crystallization did not alter considerably the fillers network, since the rheological properties were measured in the molten state and the electrical properties were measured in the solid state.

The achievement of percolation threshold at lower GN concentrations in the case LLDPE/EVA/GN (EVAMB) materials after annealing could be probably related to potential stronger immobilization of polymer chains in the interphase region, induced by the higher fraction of interfacial GN nanoplatelets in LLDPE/EVA/GN (LLDPEMB) materials [5]. However, this assumption requires further investigation.

It can be also seen from the results of fitting that the values of the scaling factor $A$ and the critical exponent $t$ corresponding to the best fits increased considerably after annealing, supporting the idea that thermal annealing helps improving the strength and the spatial distribution of conductive particle networks. Moreover, in the case of LLDPE/EVA/GN (LLDPEMB) materials, the strength of the conductive networks, reflected by the scaling factor $A$, seems to be higher while the critical exponent is higher in the case of LLDPE/EVA/GN (EVAMB) materials indicating that in this case the established conductive networks are more dependent on the filler loading.

Figure 11: Conductivity vs. $(\Phi-\Phi c)$ plots related to LLDPE/EVA/GN composites: (a) before annealing and (b) after annealing, best Power law fit and parameters are represented in each case

Compared to literature, it is worth noting that correlation between rheological and electrical percolations has been reported in some cases [20] while in other studies rheological percolation threshold has been observed for smaller concentrations [58]. The discrepancy was attributed to the different network densities required for electron transportation and stress bearing mechanisms and also to the fact that electrical measurements were conducted in the solid state while rheological measurements were done in the molten state. Similarly to rheological percolation threshold, the electrical percolation threshold values estimated in this work are higher than those reported in several studies $[12,19,27,28,58,68]$. However, they are lower than percolation concentrations achieved in most nanocomposites prepared by melt blending or containing chemically non-modified GN nanoplatelets, which is the case in this study [5, 11, 12, 19-21]. It is worth noting that the commercial GN nanoplatelets used in this work have higher average thickness and lateral size distribution as well as lower purity compared to several research-grade GN nanoplatelets.

Regarding the critical exponent corresponding to electrical percolation, values estimated for LLDPE/EVA/GN (LLDPEMB) and LLDPE/EVA/GN (EVAMB) materials, respectively equal to 3.88 and 5.2, are both higher than the universal values predicted by the percolation theory [47, $58]$ and reported in several studies regarding GN filled nanocomposites such as the recent work of Macosko et al. [27] $(t=1.73)$. Nevertheless, high values of the critical exponent $t$, in the same range of those estimated in this work, have been also reported in the literature for different GN nanocomposites [5, 24, 58, 68]. In particular, these high values were attributed to several factors, mainly the high aspect ratio of the plate-like fillers [58,70]. Besides, compared to the values of $t$ 
related to rheological percolation, the values corresponding to electrical percolation are higher. This most likely indicates that electrical conductivity above the percolation threshold is more dependent on the filler loading and the achievement of tridimensional networks.

\section{Conclusion}

In this study, we investigated a co-continuous blend containing interfacial GN nanoplatelets. Two commodity polymers, LLDPE and EVA, were used as the blend components and a commercial GN grade was selected as filler. The investigation of the rheological and electrical properties of this blend, prepared by a simple melt compounding technique, has shown good correlation between the rheological and electrical percolation thresholds $\left(\Phi_{c}\right)$. However, the networks electrical conductivity is more sensitive to GN content, compared to the networks elasticity. The estimated $\Phi_{\mathrm{c}}$ for electrical percolation is around $0.5 \mathrm{vol} \%$, which is lower than what is reported for most nanocomposites prepared by melt blending and without chemical modification. The assessment of the dynamic percolation of the blend system, through thermal annealing, revealed that both rheological and electrical networks evolve at the same timescale, with major strengthening occurring during the first 30 minutes of annealing followed by quasi-steady state. This strengthening was related to redistribution of GN networks and their migration to the interface accompanied by coarsening of the blend morphology during the early stage of annealing as well. This fast stabilization was not achieved in the case of LLDPE/GN and EVA/GN singlephase composites. Interestingly, the measurements of rheological quantities were demonstrated to be a useful tool to follow up structural changes in the filled blends during annealing, i.e. morphology changes and/or particle networks rearrangements and predict the onset of percolation. Finally, the viscoelastic response of this complex non-model system was successfully described by a two-phase model, inferring that the elastic contribution is mostly governed by GN networks while the viscous contribution is governed by the polymer matrix, regardless of its microstructure. Nevertheless, the multiphase feature of the blend matrix affects the strength of the formed networks and their space arrangement.

\section{Supplementary Material}

Viscosity data, additional TEM images and scaling parameters of the two-phase model are reported in the Electronic Supporting Information File (ESI).

\section{Acknowledgements}

The authors wish to thank MITACS, Natural Sciences and Engineering Research Council of Canada (NSERC), Fonds de recherche du Québec - Nature et technologies (FRQNT), and École de Technologie Supérieure (ÉTS) for financial support. The help of Mr. Bastien Vasseur in viscosity measurements by capillary rheometer and Mrs. Daria Strugova in extrusion is highly appreciated. 


\section{References}

[1] Kuester, S., N.R. Demarquette, J.C. Ferreira, B.G. Soares, and G.M.O. Barra, "Hybrid nanocomposites of thermoplastic elastomer and carbon nanoadditives for electromagnetic shielding“. European Polymer Journal, (2017). 88: p. 328-339.

[2] Kazemi, Y., A. Ramezani Kakroodi, A. Ameli, T. Filleter, and C.B. Park, "Highly stretchable conductive thermoplastic vulcanizate/carbon nanotube nanocomposites with segregated structure, low percolation threshold and improved cyclic electromechanical performance“. Journal of Materials Chemistry C, (2018). 6(2): p. 350-359.

[3] Zhang, K., H.-O. Yu, Y.-D. Shi, Y.-F. Chen, J.-B. Zeng, J. Guo, B. Wang, Z. Guo, and M. Wang, "Morphological regulation improved electrical conductivity and electromagnetic interference shielding in poly(l-lactide)/poly(E-caprolactone)/carbon nanotube nanocomposites via constructing stereocomplex crystallites". Journal of Materials Chemistry C, (2017). 5(11): p. 2807-2817.

[4] Jia, L.-C., D.-X. Yan, C.-H. Cui, X. Ji, and Z.-M. Li, "A Unique Double Percolated Polymer Composite for Highly Efficient Electromagnetic Interference Shielding“. Macromolecular Materials and Engineering, (2016). 301(10): p. 1232-1241.

[5] Zakiyan, S.E., H. Azizi, and I. Ghasemi, "Influence of chain mobility on rheological, dielectric and electromagnetic interference shielding properties of poly methylmethacrylate composites filled with graphene and carbon nanotube". Composites Science and Technology, (2017). 142: p. 10-19.

[6] Pang, H., L. Xu, D.-X. Yan, and Z.-M. Li, "Conductive polymer composites with segregated structures". Progress in Polymer Science, (2014). 39(11): p. 1908-1933.

[7] Burns, N.M., R.M. Eichhorn, and C.G. Reid, "Stress controlling semiconductive shields in medium voltage power distribution cables". IEEE Electrical Insulation Magazine, (1992). 8(5): p. 8-24.

[8] Fritjof, N. and U. Mikael, "Conductivity simulations of field-grading composites". Journal of Physics D: Applied Physics, (2016). 49(33): p. 335303.

[9] Gaska, K., X. Xu, S. Gubanski, and R. Kádár, "Electrical, Mechanical, and Thermal Properties of LDPE Graphene Nanoplatelets Composites Produced by Means of Melt Extrusion Process". Polymers, (2017). 9(1): p. 11.

[10] Mittal, G., V. Dhand, K.Y. Rhee, S.-J. Park, and W.R. Lee, "A review on carbon nanotubes and graphene as fillers in reinforced polymer nanocomposites". Journal of Industrial and Engineering Chemistry, (2015). 21: p. 11-25.

[11] Kim, H., A.A. Abdala, and C.W. Macosko, "Graphene/Polymer Nanocomposites". Macromolecules, (2010). 43(16): p. 6515-6530.

[12] Kuilla, T., S. Bhadra, D. Yao, N.H. Kim, S. Bose, and J.H. Lee, "Recent advances in graphene based polymer composites“. Progress in Polymer Science, (2010). 35(11): p. 1350-1375.

[13] Kim, H. and C.W. Macosko, "Morphology and Properties of Polyester/Exfoliated Graphite Nanocomposites". Macromolecules, (2008). 41(9): p. 3317-3327.

[14] Zhu, Y., S. Murali, W. Cai, X. Li, J.W. Suk, J.R. Potts, and R.S. Ruoff, "Graphene and Graphene Oxide: Synthesis, Properties, and Applications". Advanced Materials, (2010). 22(35): p. 3906-3924.

[15] Azizi, S., E. David, M.F. Fréchette, P. Nguyen-Tri, and C.M. Ouellet-Plamondon, "Electrical and thermal phenomena in low-density polyethylene/carbon black composites near the percolation threshold“. Journal of Applied Polymer Science. 0(0): p. 47043.

[16] Kuester, S., G.M. Barra, and N.R. Demarquette, "Morphology, mechanical properties and electromagnetic shielding effectiveness of poly(styrene-b-ethylene-ran-butylene-bstyrene)/carbon nanotube nanocomposites: effects of maleic anhydride, carbon nanotube loading and processing method". Polymer International, (2018). 67(9): p. 1229-1240. 
[17] Novoselov, K.S., A.K. Geim, S.V. Morozov, D. Jiang, Y. Zhang, S.V. Dubonos, I.V. Grigorieva, and A.A. Firsov, "Electric Field Effect in Atomically Thin Carbon Films". Science, (2004). 306(5696): p. 666-669.

[18] Choi, W., I. Lahiri, R. Seelaboyina, and Y.S. Kang, "Synthesis of Graphene and Its Applications: A Review". Critical Reviews in Solid State and Materials Sciences, (2010). 35(1): p. 52-71.

[19] Tripathi, S.N., G.S.S. Rao, A.B. Mathur, and R. Jasra, "Polyolefin/graphene nanocomposites: a review". RSC Advances, (2017). 7(38): p. 23615-23632.

[20] Kim, H., S. Kobayashi, M.A. AbdurRahim, M.J. Zhang, A. Khusainova, M.A. Hillmyer, A.A. Abdala, and C.W. Macosko, "Graphene/polyethylene nanocomposites: Effect of polyethylene functionalization and blending methods". Polymer, (2011). 52(8): p. 18371846.

[21] Wang, F., Y. Zhang, B.B. Zhang, R.Y. Hong, M.R. Kumar, and C.R. Xie, "Enhanced electrical conductivity and mechanical properties of ABS/EPDM composites filled with graphene". Composites Part B: Engineering, (2015). 83: p. 66-74.

[22] Biswas, S., G.P. Kar, and S. Bose, "Tailor-Made Distribution of Nanoparticles in Blend Structure toward Outstanding Electromagnetic Interference Shielding“. ACS Applied Materials \& Interfaces, (2015). 7(45): p. 25448-25463.

[23] Saravanan, N., R. Rajasekar, S. Mahalakshmi, T.P. Sathishkumar, K.S.K. Sasikumar, and S. Sahoo, "Graphene and modified graphene-based polymer nanocomposites - A review". Journal of Reinforced Plastics and Composites, (2014). 33(12): p. 1158-1170.

[24] Liao, K.-H., Y. Qian, and C.W. Macosko, "Ultralow percolation graphene/polyurethane acrylate nanocomposites“. Polymer, (2012). 53(17): p. 3756-3761.

[25] Gao, C., S. Zhang, F. Wang, P. Liu, Y. Ding, and M. Yang, "Conductive composites with segregated structure and ultralow percolation threshold via flocculation-assembled PVDF/graphene core-shell particles". Materials Letters, (2015). 158: p. 428-431.

[26] Wang, Z., X. Shen, N.M. Han, X. Liu, Y. Wu, W. Ye, and J.-K. Kim, "Ultralow Electrical Percolation in Graphene Aerogel/Epoxy Composites". Chemistry of Materials, (2016). 28(18): p. 6731-6741.

[27] Bai, L., S. He, J.W. Fruehwirth, A. Stein, C.W. Macosko, and X. Cheng, "Localizing graphene at the interface of cocontinuous polymer blends: Morphology, rheology, and conductivity of cocontinuous conductive polymer composites". Journal of Rheology, (2017). 61(4): p. 575-587.

[28] Bai, L., R. Sharma, X. Cheng, and C.W. Macosko, "Kinetic Control of Graphene Localization in Co-continuous Polymer Blends via Melt Compounding". Langmuir, (2018). 34(3): p. 1073-1083.

[29] Kurusu, R.S., E. Helal, N. Moghimian, E. David, and N. Demarquette, "The Role of Selectively Located Commercial Graphene Nanoplatelets in the Electrical Properties, Morphology, and Stability of EVA/LLDPE Blends". Macromolecular Materials and Engineering, (2018). 303(9): p. 1800187.

[30] Scherzer, S.L., E. Pavlova, J.D. Esper, and Z. Starý, "Phase structure, rheology and electrical conductivity of co-continuous polystyrene/polymethylmethacrylate blends filled with carbon black". Composites Science and Technology, (2015). 119: p. 138-147.

[31] Otero-Navas, I., M. Arjmand, and U. Sundararaj, "Carbon nanotube induced double percolation in polymer blends: Morphology, rheology and broadband dielectric properties“. Polymer, (2017). 114: p. 122-134.

[32] Soares, B.G., F. Touchaleaume, L.F. Calheiros, and G.M.O. Barra, "Effect of double percolation on the electrical properties and electromagnetic interference shielding effectiveness of carbon-black-loaded polystyrenelethylene vinyl acetate copolymer blends“. Journal of Applied Polymer Science, (2016). 133(7). 
[33] Sumita, M., K. Sakata, Y. Hayakawa, S. Asai, K. Miyasaka, and M. Tanemura, "Double percolation effect on the electrical conductivity of conductive particles filled polymer blends". Colloid and Polymer Science, (1992). 270(2): p. 134-139.

[34] Gubbels, F., S. Blacher, E. Vanlathem, R. Jerome, R. Deltour, F. Brouers, and P. Teyssie, "Design of Electrical Composites: Determining the Role of the Morphology on the Electrical Properties of Carbon Black Filled Polymer Blends". Macromolecules, (1995). 28(5): p. 1559-1566.

[35] Gubbels, F., R. Jerome, E. Vanlathem, R. Deltour, S. Blacher, and F. Brouers, "Kinetic and Thermodynamic Control of the Selective Localization of Carbon Black at the Interface of Immiscible Polymer Blends". Chemistry of Materials, (1998). 10(5): p. 12271235.

[36] Zhao, X., J. Zhao, J.-P. Cao, X. Wang, M. Chen, and Z.-M. Dang, "Tuning the Dielectric Properties of Polystyrene/Poly(vinylidene fluoride) Blends by Selectively Localizing Carbon Black Nanoparticles". The Journal of Physical Chemistry B, (2013). 117(8): p. 2505-2515.

[37] Gubbels, F., R. Jerome, P. Teyssie, E. Vanlathem, R. Deltour, A. Calderone, V. Parente, and J.L. Bredas, "Selective Localization of Carbon Black in Immiscible Polymer Blends: A Useful Tool To Design Electrical Conductive Composites". Macromolecules, (1994). 27(7): p. 1972-1974.

[38] Huang, S., L. Bai, M. Trifkovic, X. Cheng, and C.W. Macosko, "Controlling the Morphology of Immiscible Cocontinuous Polymer Blends via Silica Nanoparticles Jammed at the Interface". Macromolecules, (2016). 49(10): p. 3911-3918.

[39] Shen, Y., T.-T. Zhang, J.-H. Yang, N. Zhang, T. Huang, and Y. Wang, "Selective localization of reduced graphene oxides at the interface of PLA/EVA blend and its resultant electrical resistivity“. Polymer Composites, (2017). 38(9): p. 1982-1991.

[40] Tan, Y., L. Fang, J. Xiao, Y. Song, and Q. Zheng, "Grafting of copolymers onto graphene by miniemulsion polymerization for conductive polymer composites: improved electrical conductivity and compatibility induced by interfacial distribution of graphene". Polymer Chemistry, (2013). 4(10): p. 2939-2944.

[41] Genoyer, J., M. Yee, J. Soulestin, and N. Demarquette, "Compatibilization mechanism induced by organoclay in PMMA/PS blends". Journal of Rheology, (2017). 61(4): p. 613626.

[42] Salzano de Luna, M. and G. Filippone, "Effects of nanoparticles on the morphology of immiscible polymer blends - Challenges and opportunities". European Polymer Journal, (2016). 79: p. 198-218.

[43] Helal, E., C. Pottier, E. David, M. Fréchette, and N.R. Demarquette, "Polyethylene/thermoplastic elastomer/Zinc Oxide nanocomposites for high voltage insulation applications: Dielectric, mechanical and rheological behavior". European Polymer Journal, (2018). 100: p. 258-269.

[44] Trifkovic, M., A.T. Hedegaard, M. Sheikhzadeh, S. Huang, and C.W. Macosko, "Stabilization of PE/PEO Cocontinuous Blends by Interfacial Nanoclays". Macromolecules, (2015). 48(13): p. 4631-4644.

[45] Vandebril, S., J. Vermant, and P. Moldenaers, "Efficiently suppressing coalescence in polymer blends using nanoparticles: role of interfacial rheology". Soft Matter, (2010). 6(14): p. 3353-3362.

[46] Parameswaranpillai, J.E., Hameed, N. (Ed.), Kurian, T. (Ed.), Yu, Y. (Ed.). (2017), “ Nanocomposite Materials Synthesis, Properties and Applications (Demarquette, N., Carastan, D., Chapter 10: Rheological behavior of nanocomposites)“. CRC Press., (2017). 
[47] Clerc, J.P., G. Giraud, J.M. Laugier, and J.M. Luck, "The electrical conductivity of binary disordered systems, percolation clusters, fractals and related models". Advances in Physics, (1990). 39(3): p. 191-309.

[48] Filippone, G., G. Romeo, and D. Acierno, "Viscoelasticity and Structure of Polystyrene/Fumed Silica Nanocomposites: Filler Network and Hydrodynamic Contributions". Langmuir, (2010). 26(4): p. 2714-2720.

[49] Filippone, G. and M. Salzano de Luna, "A Unifying Approach for the Linear Viscoelasticity of Polymer Nanocomposites". Macromolecules, (2012). 45(21): p. 88538860.

[50] Filippone, G., M. Salzano de Luna, D. Acierno, and P. Russo, "Elasticity and structure of weak graphite nanoplatelet (GNP) networks in polymer matrices through viscoelastic analyses". Polymer, (2012). 53(13): p. 2699-2704.

[51] Romeo, G., G. Filippone, P. Russo, and D. Acierno, "Effects of particle dimension and matrix viscosity on the colloidal aggregation in weakly interacting polymer-nanoparticle composites: a linear viscoelastic analysis“. Polymer Bulletin, (2009). 63(6): p. 883.

[52] Altobelli, R., M. Salzano de Luna, and G. Filippone, "Interfacial crowding of nanoplatelets in co-continuous polymer blends: assembly, elasticity and structure of the interfacial nanoparticle network". Soft Matter, (2017). 13(37): p. 6465-6473.

[53] Filippone, G., A. Causa, M. Salzano de Luna, L. Sanguigno, and D. Acierno, "Assembly of plate-like nanoparticles in immiscible polymer blends - effect of the presence of a preferred liquid-liquid interface“. Soft Matter, (2014). 10(18): p. 3183-3191.

[54] Li, W., Y. Zhang, J. Yang, J. Zhang, Y. Niu, and Z. Wang, "Thermal Annealing Induced Enhancements of Electrical Conductivities and Mechanism for Multiwalled Carbon Nanotubes Filled Poly(Ethylene-co-Hexene) Composites“. ACS Applied Materials \& Interfaces, (2012). 4(12): p. 6468-6478.

[55] Zhang, C., P. Wang, C.-a. Ma, G. Wu, and M. Sumita, "Temperature and time dependence of conductive network formation: Dynamic percolation and percolation time“. Polymer, (2006). 47(1): p. 466-473.

[56] Alig, I., P. Pötschke, D. Lellinger, T. Skipa, S. Pegel, G.R. Kasaliwal, and T. Villmow, "Establishment, morphology and properties of carbon nanotube networks in polymer melts". Polymer, (2012). 53(1): p. 4-28.

[57] Huang, S., Z. Liu, C. Yin, Y. Wang, Y. Gao, C. Chen, and M. Yang, "Dynamic Electrical and Rheological Percolation in Isotactic Poly(propylene)/Carbon Black Composites". Macromolecular Materials and Engineering, (2012). 297(1): p. 51-59.

[58] Gao, C., P. Liu, Y. Ding, T. Li, F. Wang, J. Chen, S. Zhang, Z. Li, and M. Yang, "Noncontact percolation of unstable graphene networks in poly(styrene-co-acrylonitrile) nanocomposites: Electrical and rheological properties". Composites Science and Technology, (2018). 155: p. 41-49.

[59] Batista, N.L., E. Helal, R.S. Kurusu, N. Moghimian, E. David, N. Demarquette and P. Hubert, "Mass Produced Graphene - HDPE Nanocomposites: Thermal, Rheological, Electrical and Mechanical Properties". Polymer Engineering and Science, Volume59, Issue4, April 2019, p. 675-682.

[60] Moghimian, N., S. Saeidlou, H. Lentzakis, G.F. Rosi, N. Song, and D. É. Electrical conductivity of commercial graphene polyethylene nanocomposites. in 2017 IEEE 17th International Conference on Nanotechnology (IEEE-NANO). 2017.

[61] Kurusu, R.S., E. Helal, N. Moghimian, N. Demarquette and E. David, "Electrical properties of EVA/LLDPE blends with selectively located graphene nanoplatelets". 2nd IEE International Conference on Dielectrics, (July 2018).

[62] Helal, E., R.S. Kurusu., E. David, N. Moghimian and N.R. Demarquette "Use of Rheology as a Tool to Reduce the Nanoparticles Percolation Threshold in Conductive 
Nanocomposites“. 34th International Conference of the Polymer Processing Society (PPS 34), (2018).

[63] Hepperle, J., Rheological Properties of Polymer Melts, in Co-Rotating Twin-Screw Extruder. 2007, Carl Hanser Verlag GmbH \& Co. KG. p. 35-55.

[64] Jang, B.Z. and A. Zhamu, "Processing of nanographene platelets (NGPS) and NGP nanocomposites: a review“. Journal of Materials Science, (2008). 43(15): p. 5092-5101.

[65] G. M. Jordhamo, J.A.M., L. H. Sperling, " Phase continuity and inversion in polymer blends and simultaneous interpenetrating networks". Polymer Engineering \& Science, (1986). 26(8): p. 517-524.

[66] Göldel, A., G.R. Kasaliwal, P. Pötschke, and G. Heinrich, "The kinetics of CNT transfer between immiscible blend phases during melt mixing“. Polymer, (2012). 53(2): p. 411421.

[67] Göldel, A., A. Marmur, G.R. Kasaliwal, P. Pötschke, and G. Heinrich, "Shape-Dependent Localization of Carbon Nanotubes and Carbon Black in an Immiscible Polymer Blend during Melt Mixing“. Macromolecules, (2011). 44(15): p. 6094-6102.

[68] Gao, C., S. Zhang, F. Wang, B. Wen, C. Han, Y. Ding, and M. Yang, "Graphene Networks with Low Percolation Threshold in ABS Nanocomposites: Selective Localization and Electrical and Rheological Properties". ACS Applied Materials \& Interfaces, (2014). 6(15): p. 12252-12260.

[69] Arbabi, S. and M. Sahimi, "Mechanics of disordered solids. I. Percolation on elastic networks with central forces“. Physical Review B, (1993). 47(2): p. 695-702.

[70] Mamunya, Y.P., V.V. Davydenko, P. Pissis, and E.V. Lebedev, "Electrical and thermal conductivity of polymers filled with metal powders". European Polymer Journal, (2002). 38(9): p. 1887-1897. 
\begin{tabular}{l|l} 
(c) & (d)
\end{tabular}

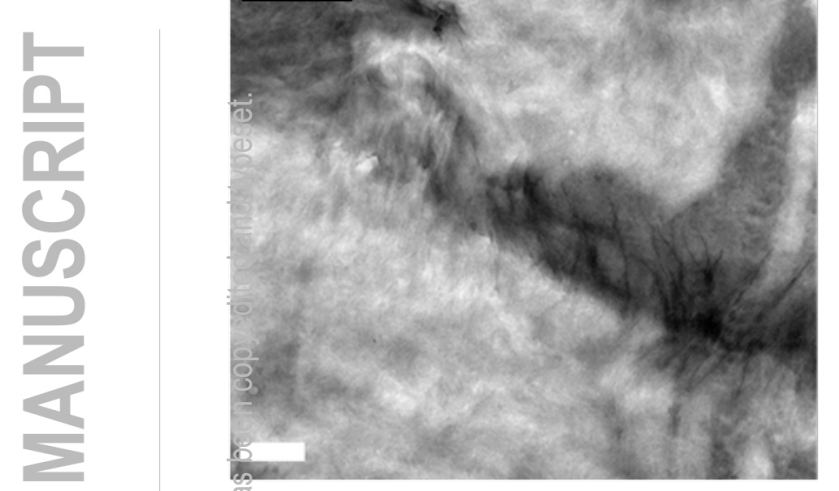

0
$\frac{1}{10}$
$\frac{1}{4}$
4

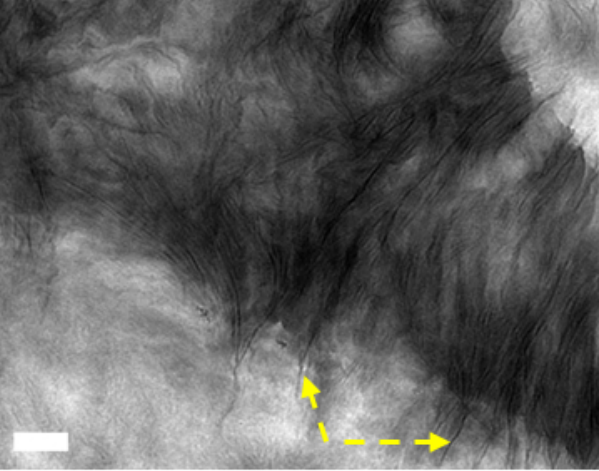

8

訔 웅

ఖ

言 눙

은 공

융

은

든

变

(1)

틀

듬

这

핱

오 쁨

흥어

㟧

뜰

믐

8

D 


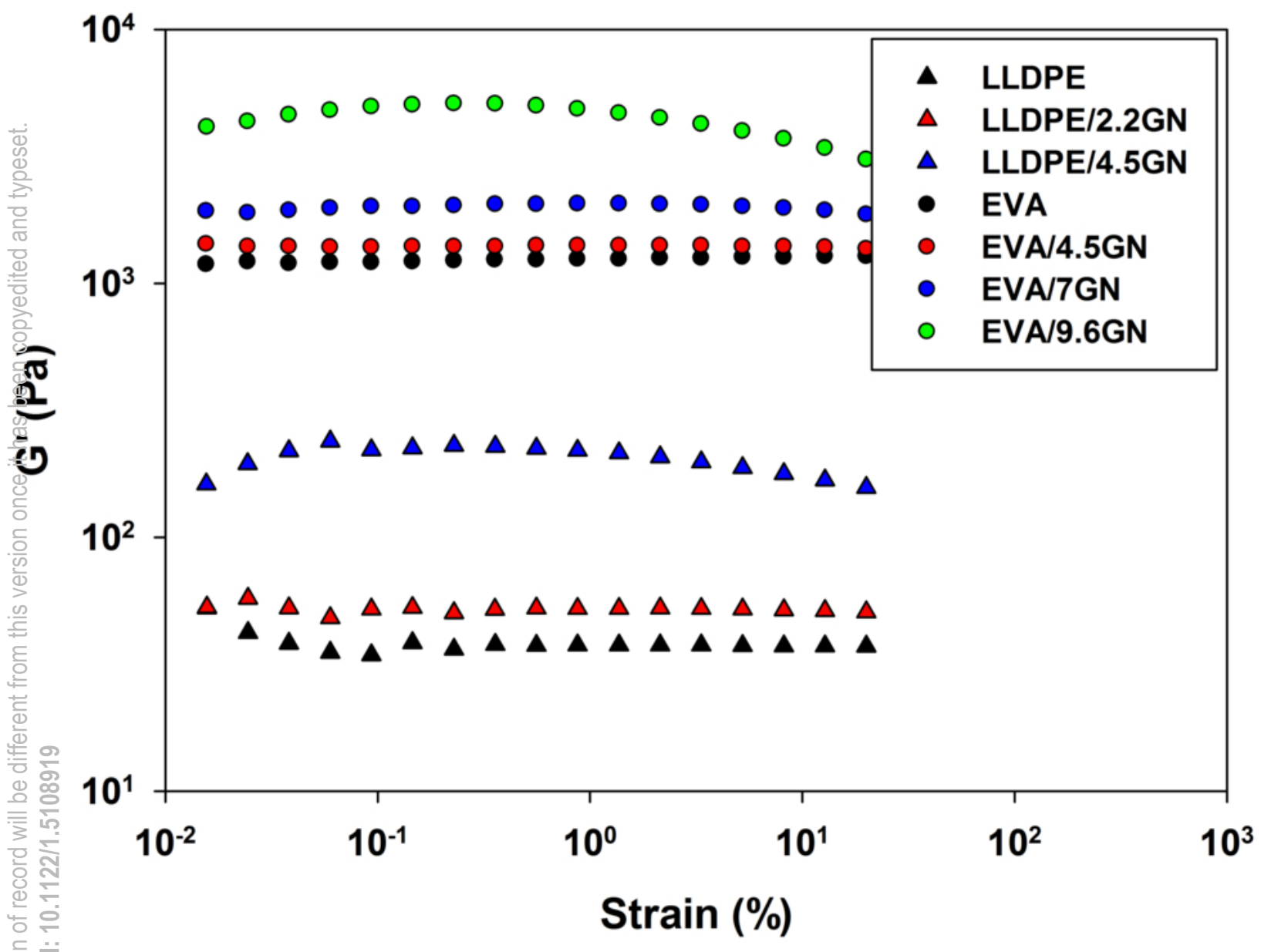




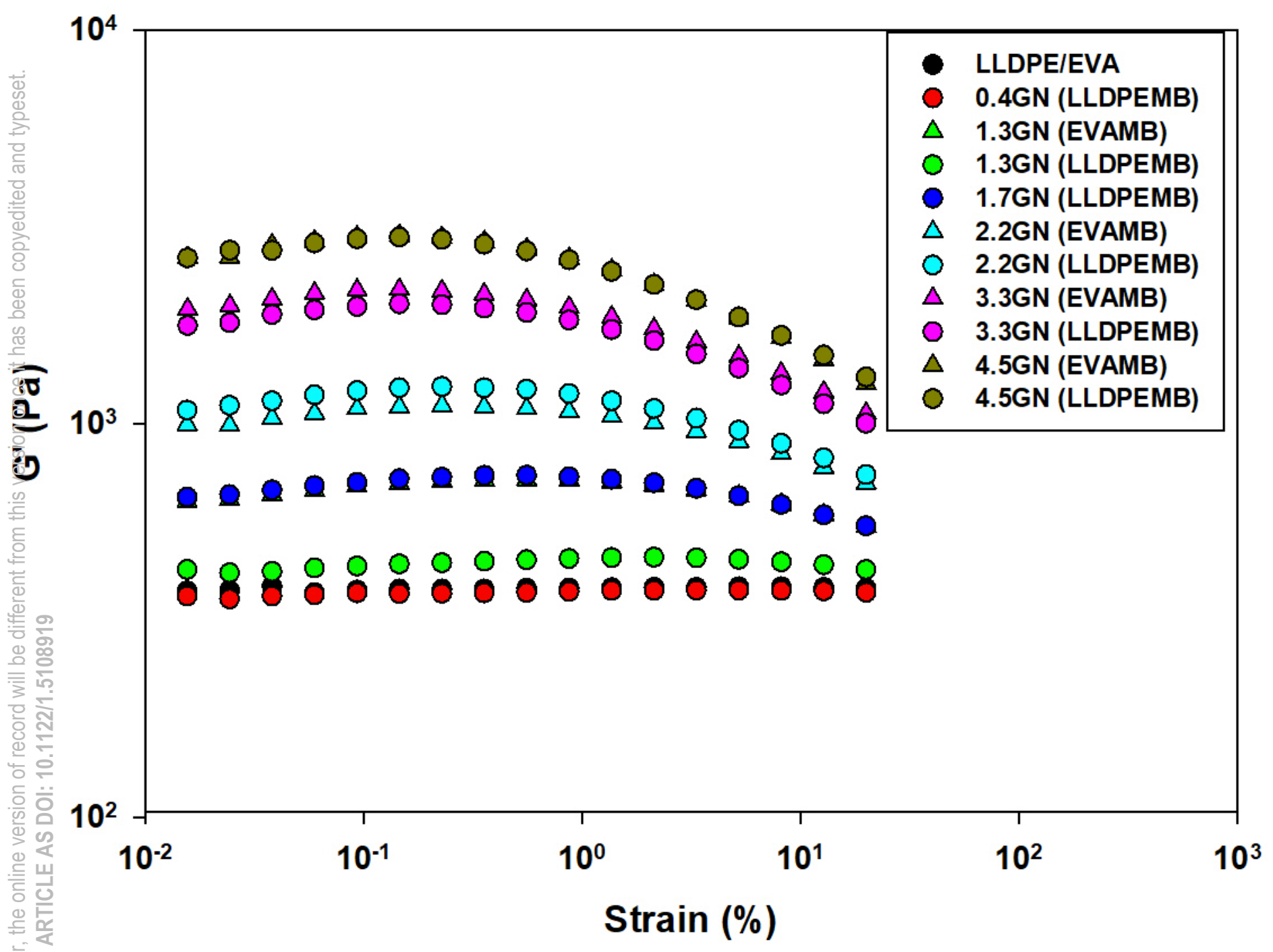




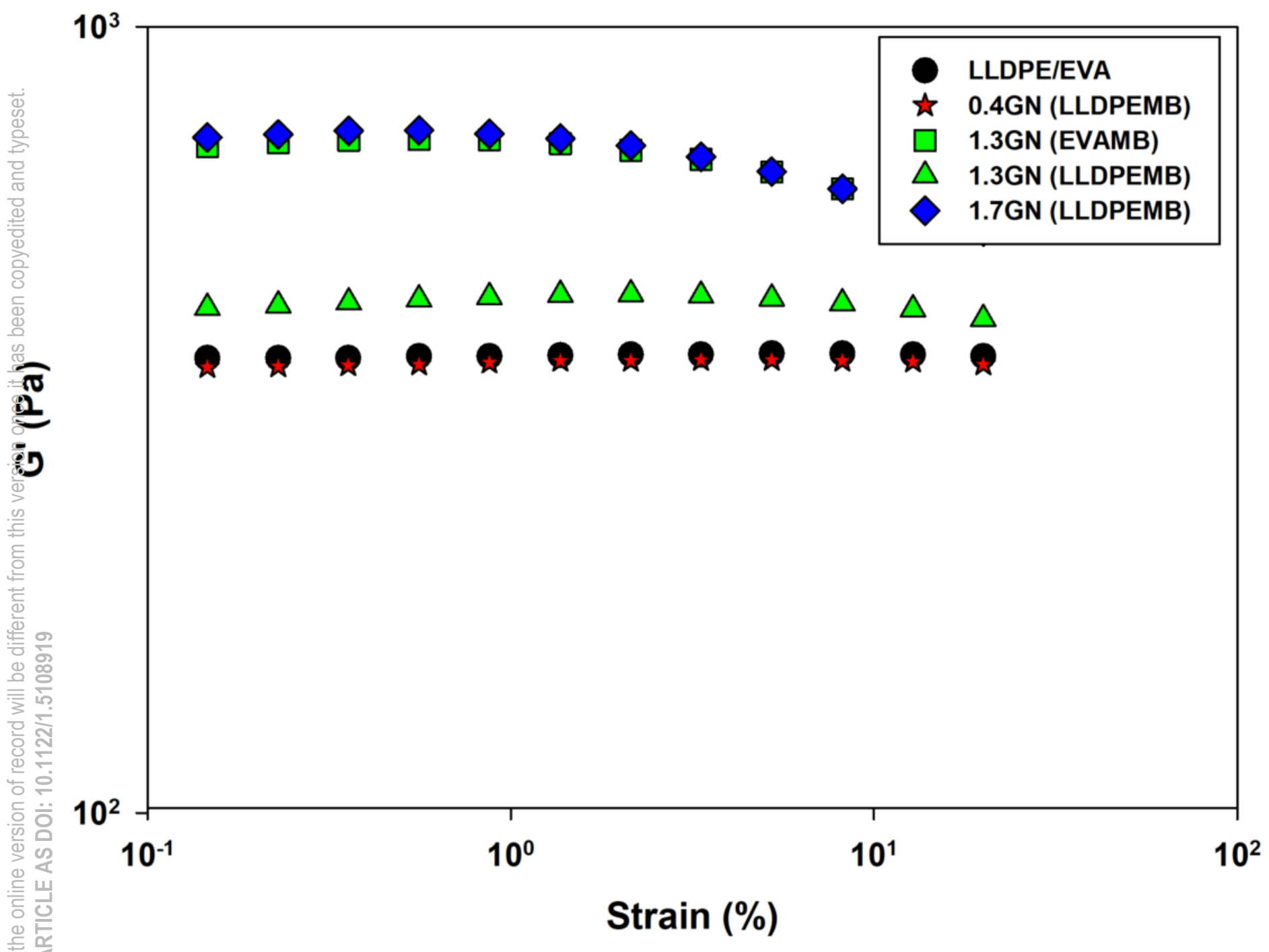




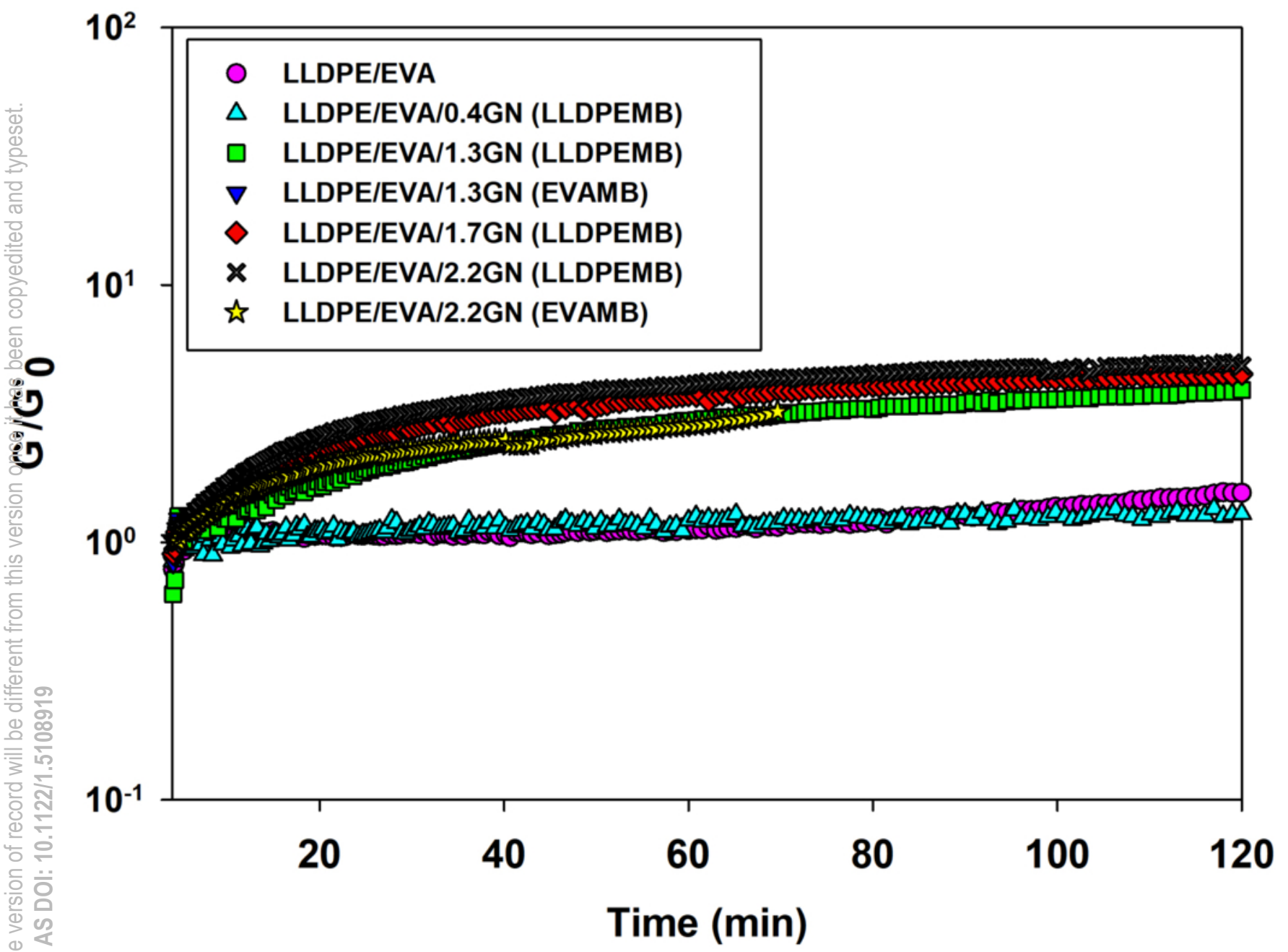




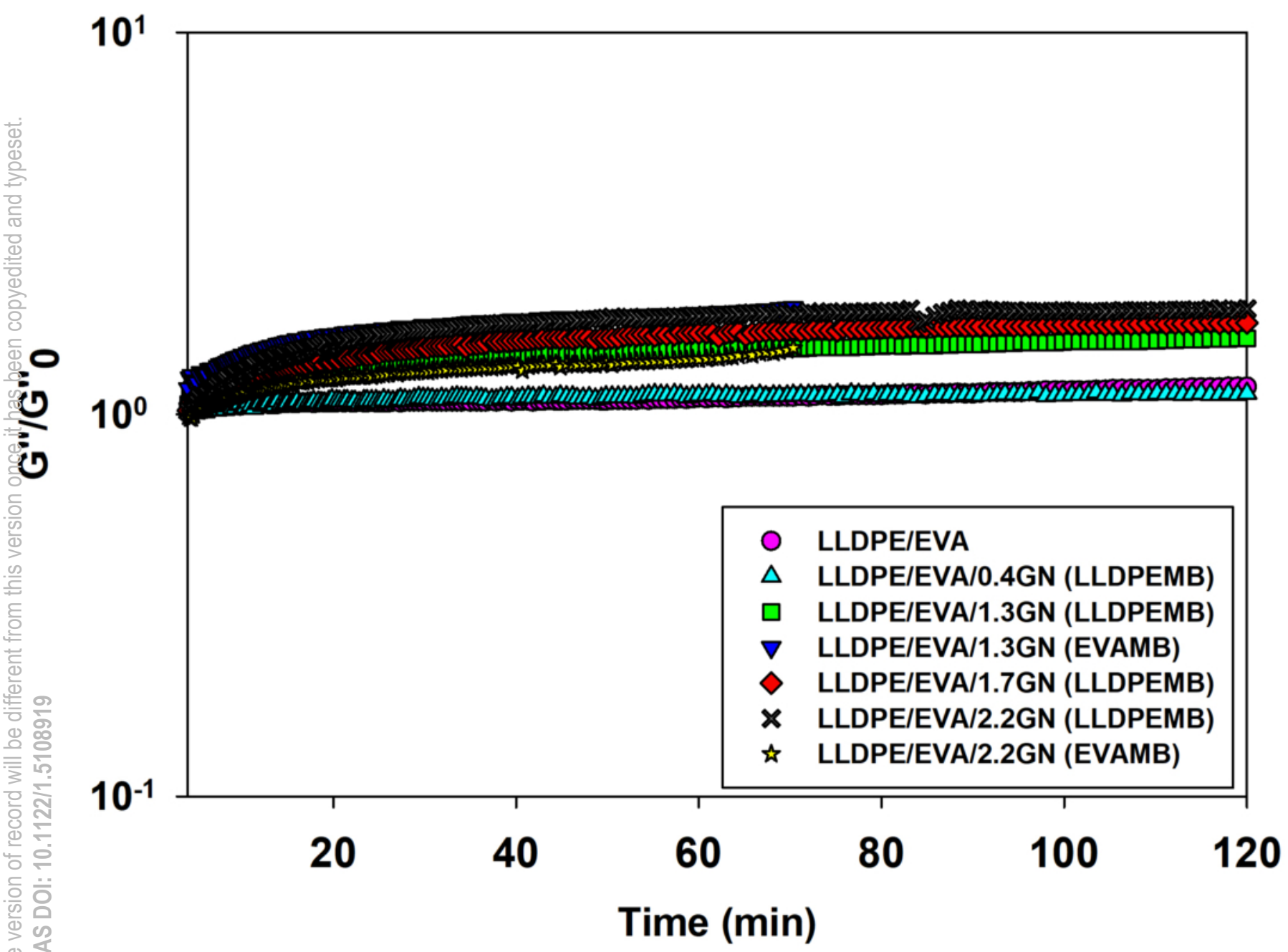




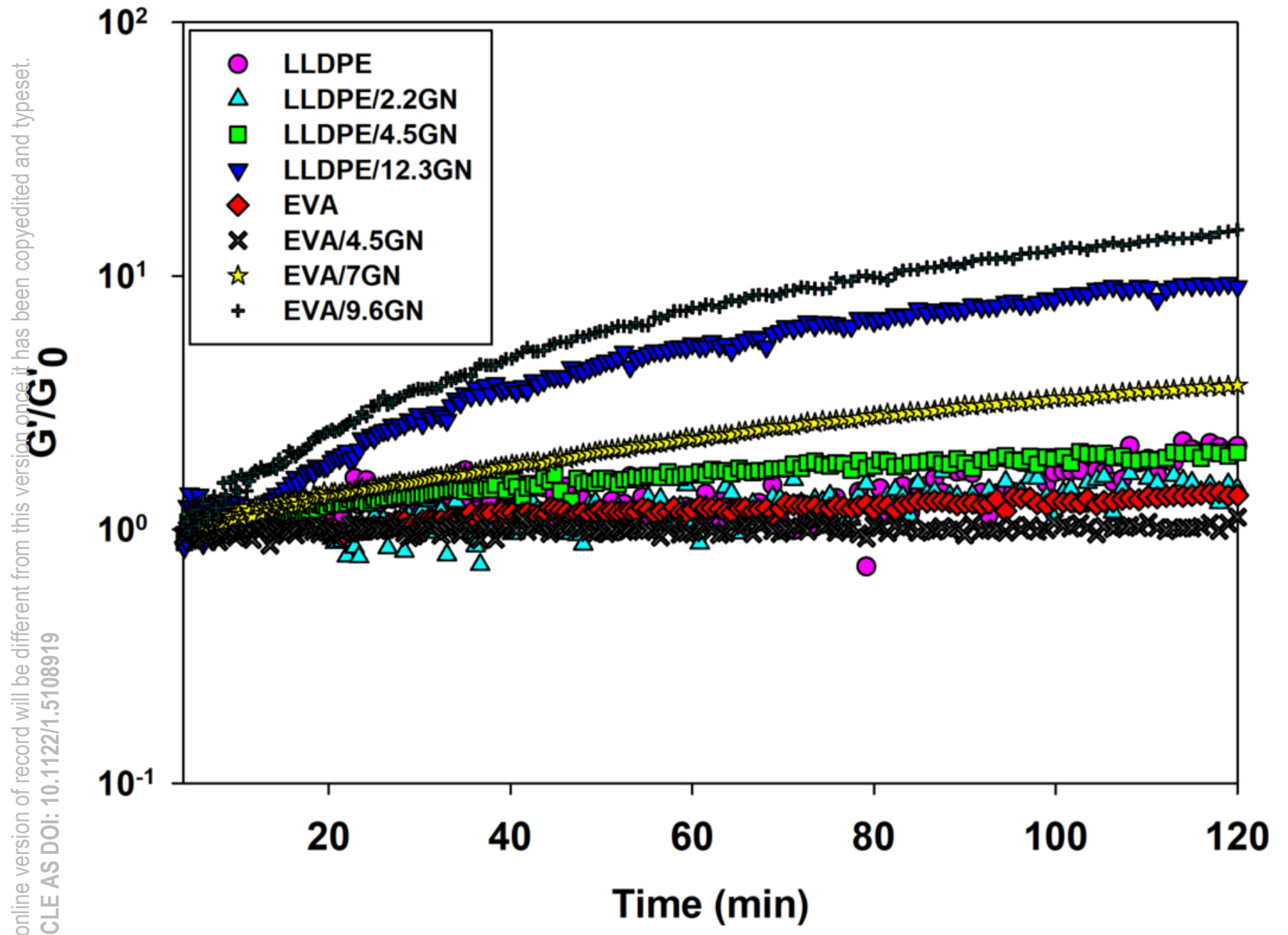




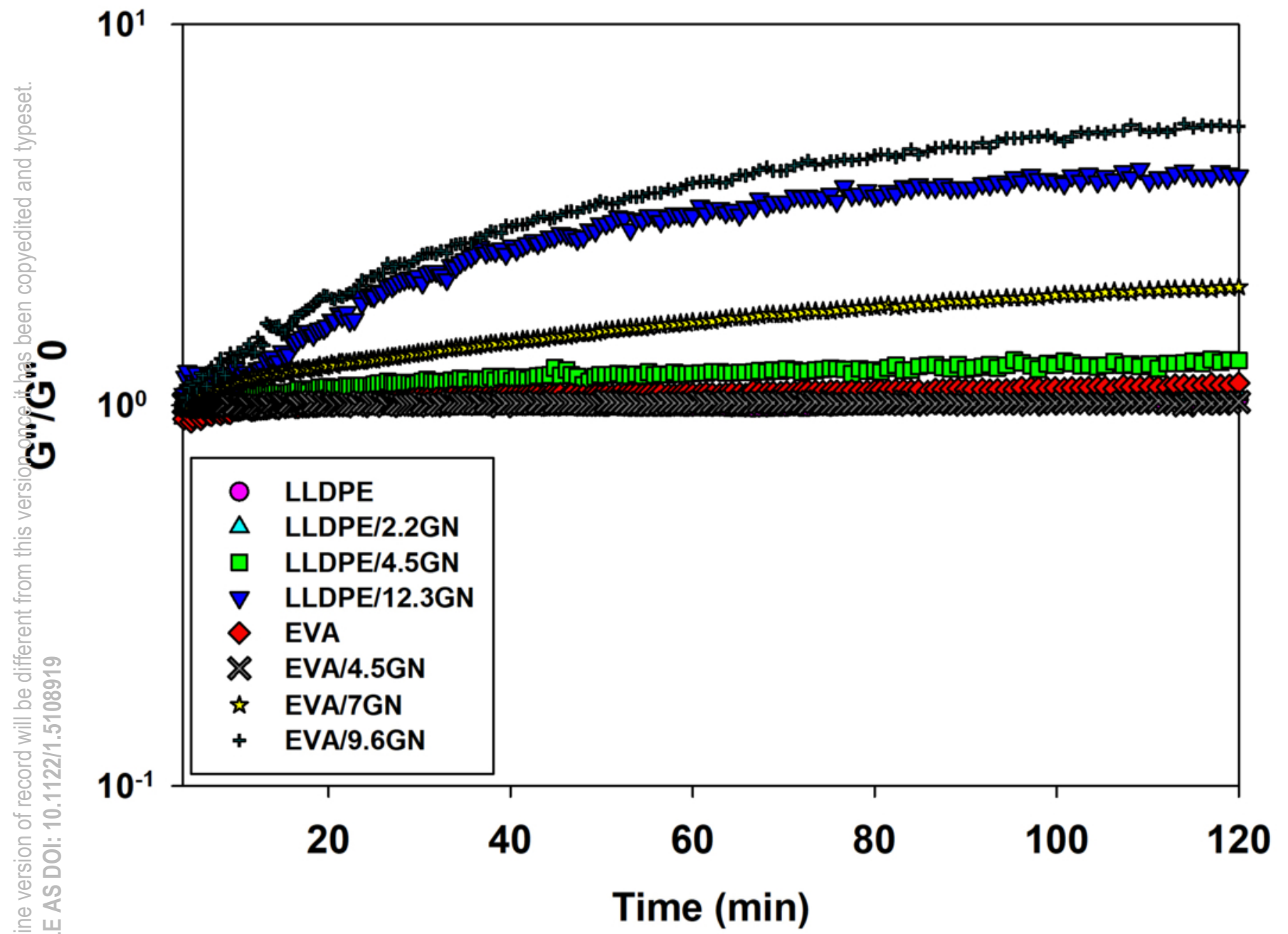


Annealing at $160^{\circ} \mathrm{C}$

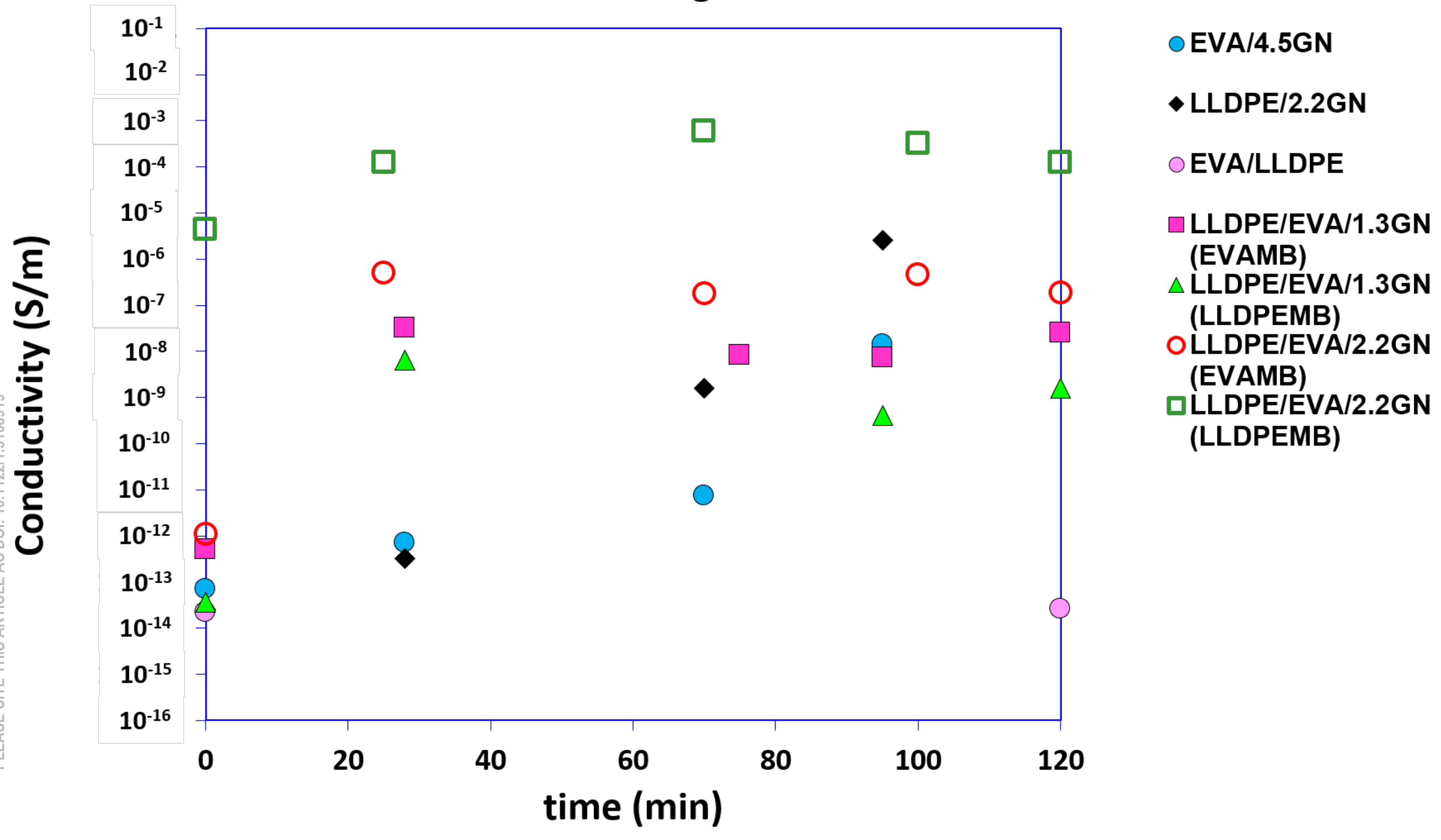




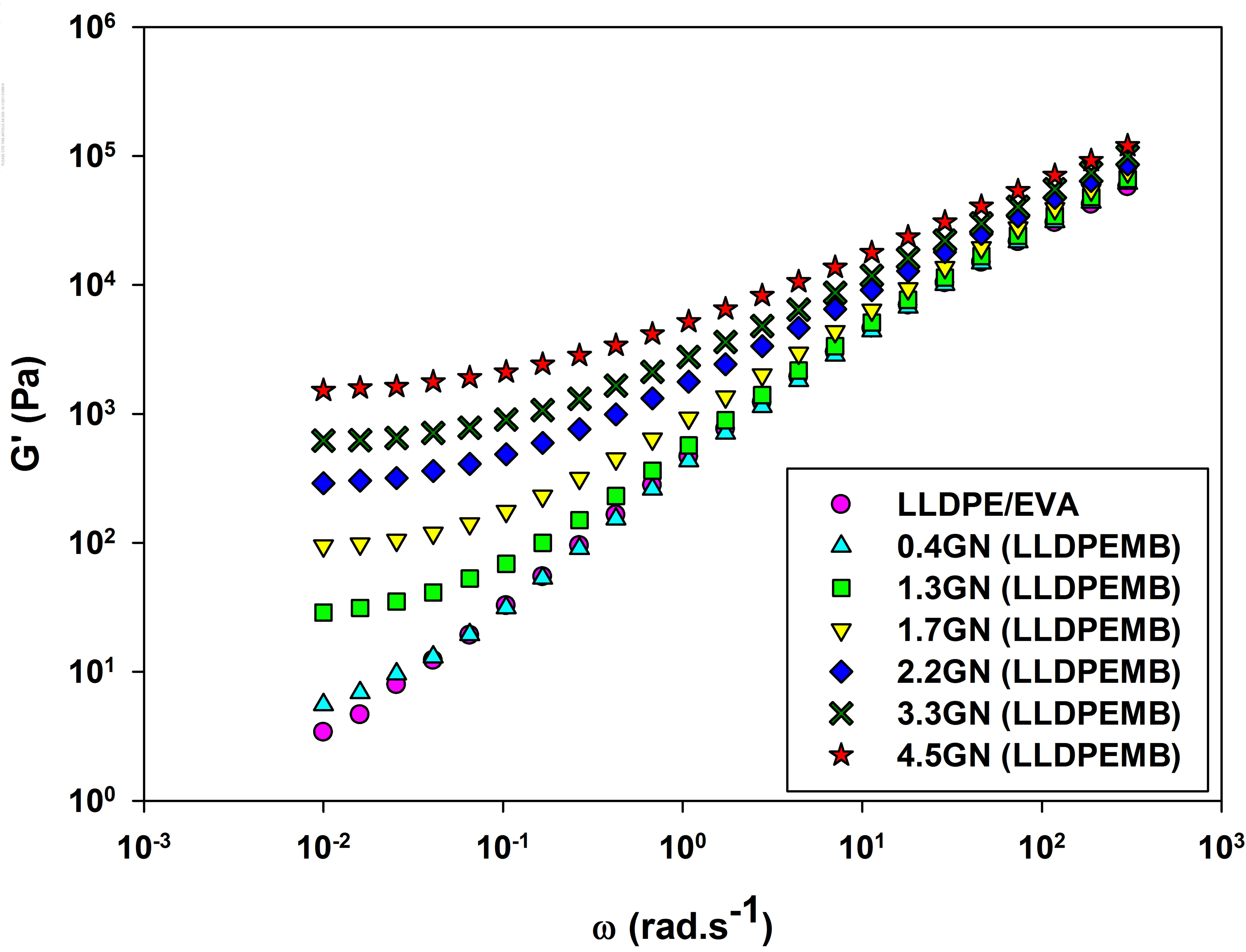




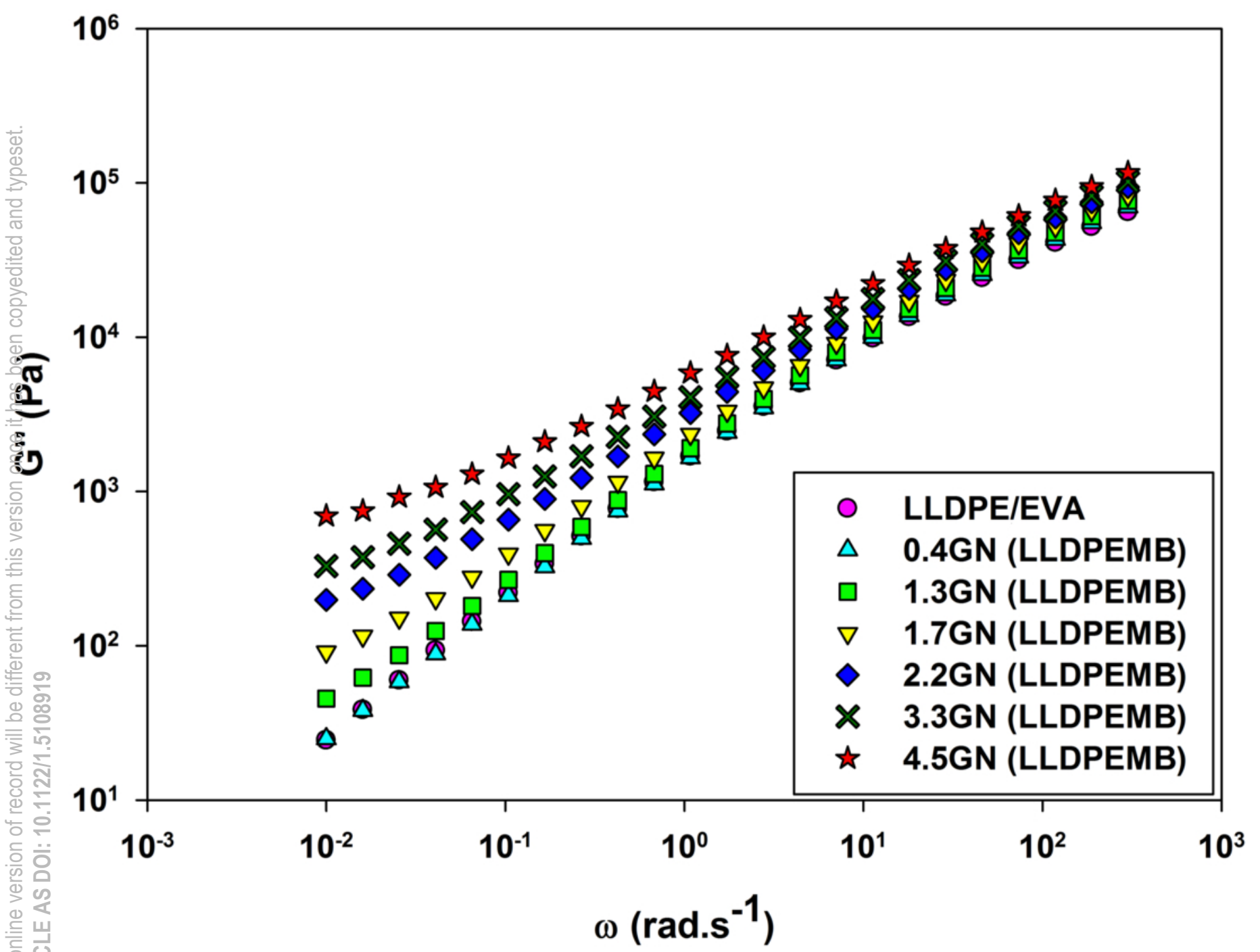




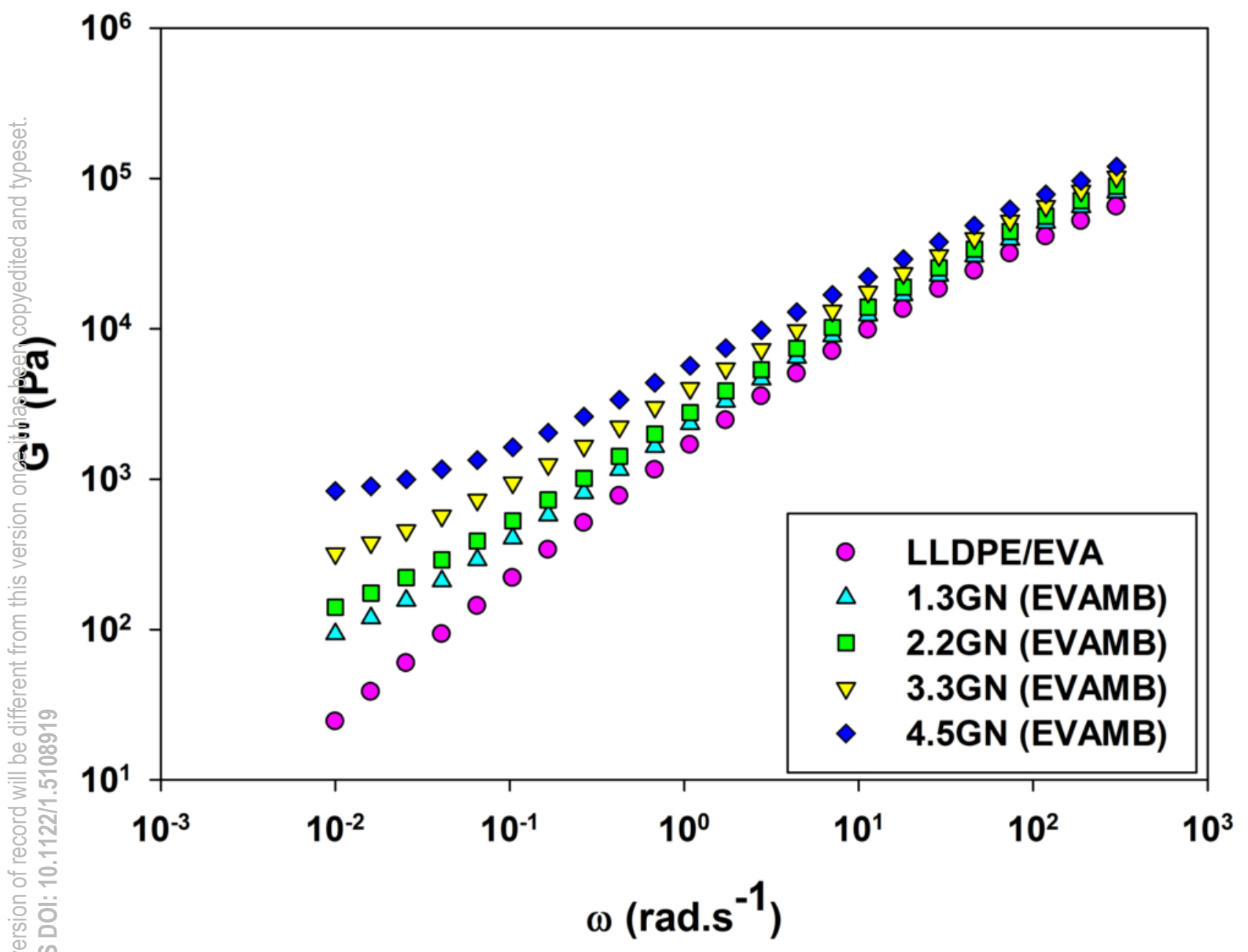




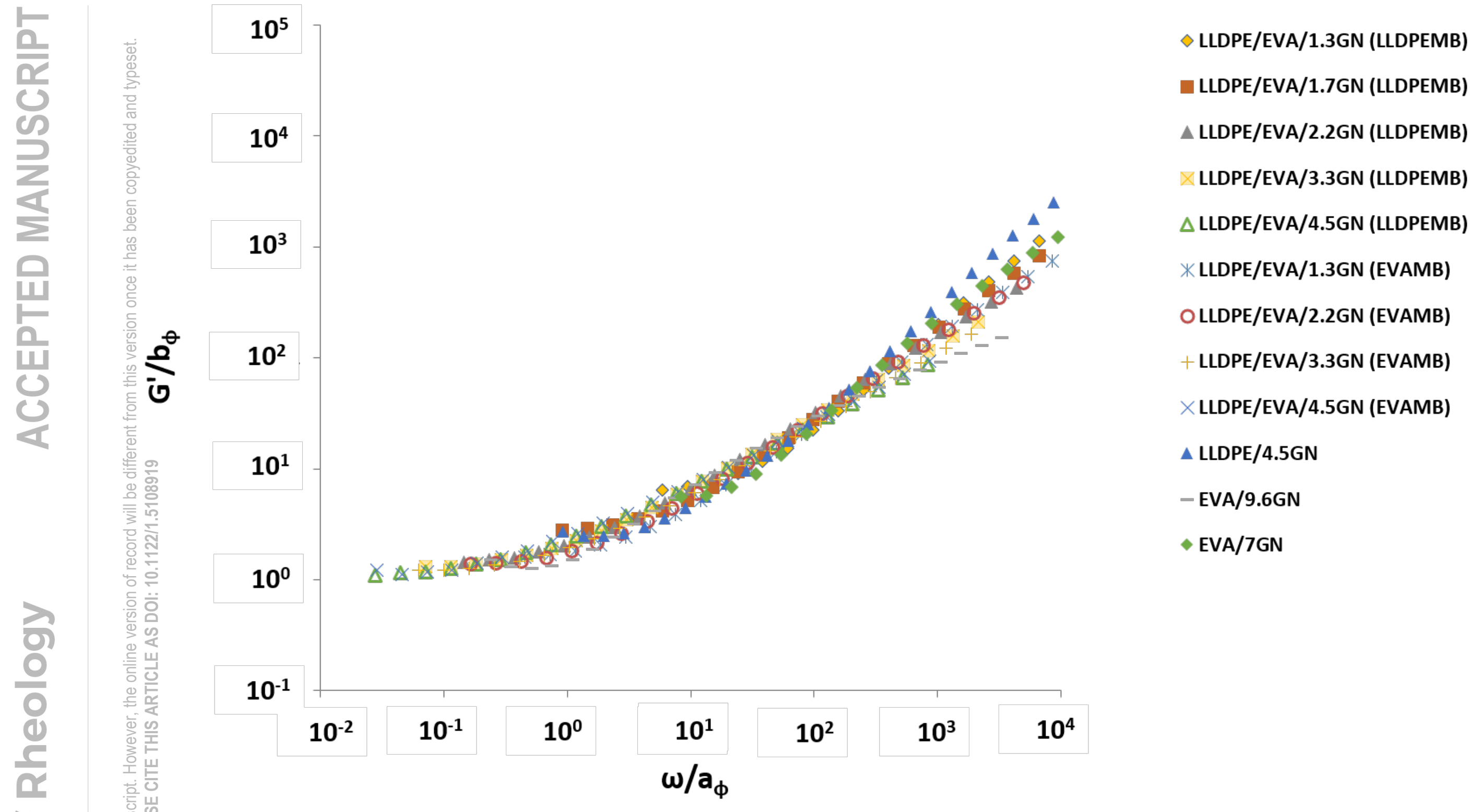




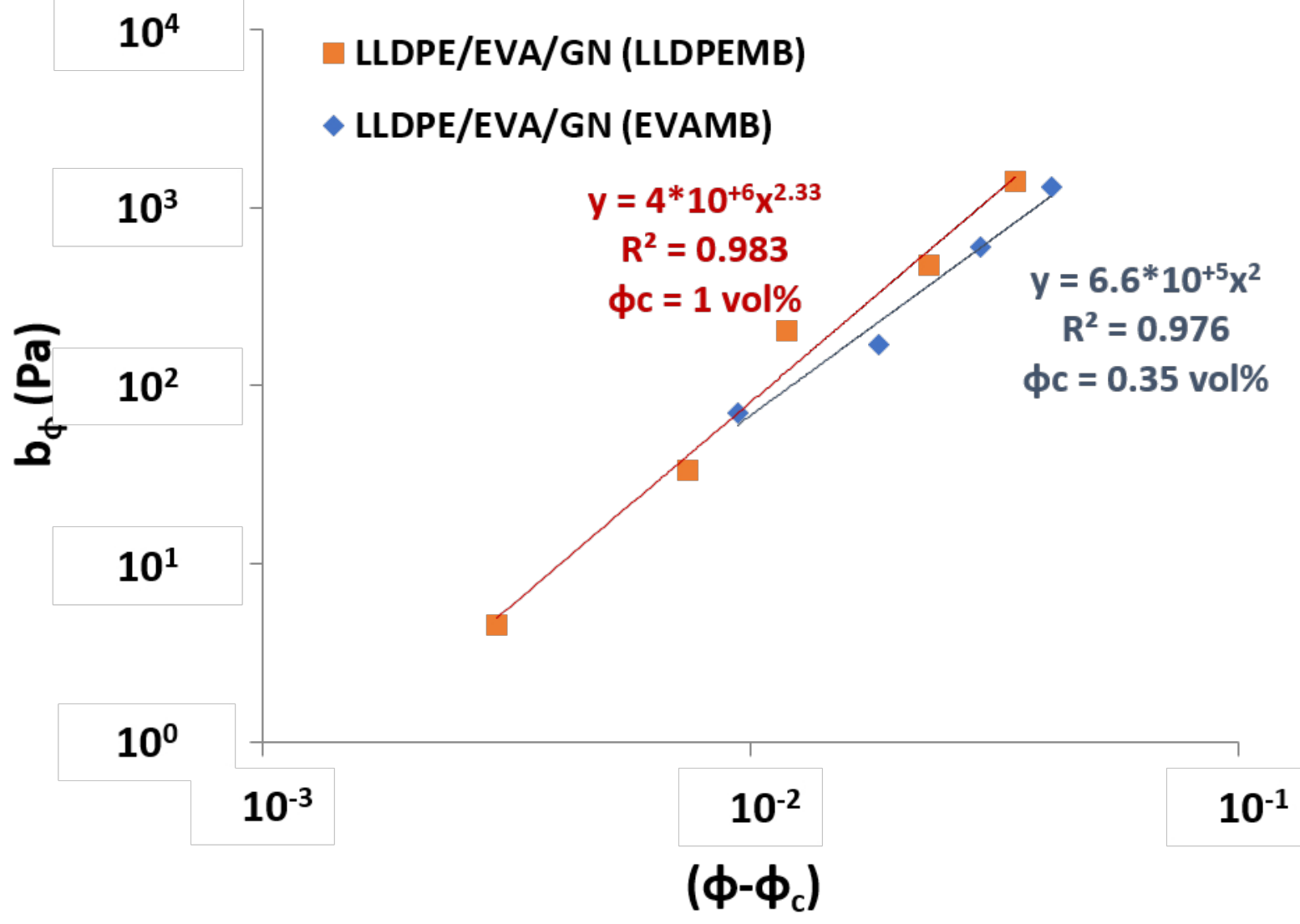




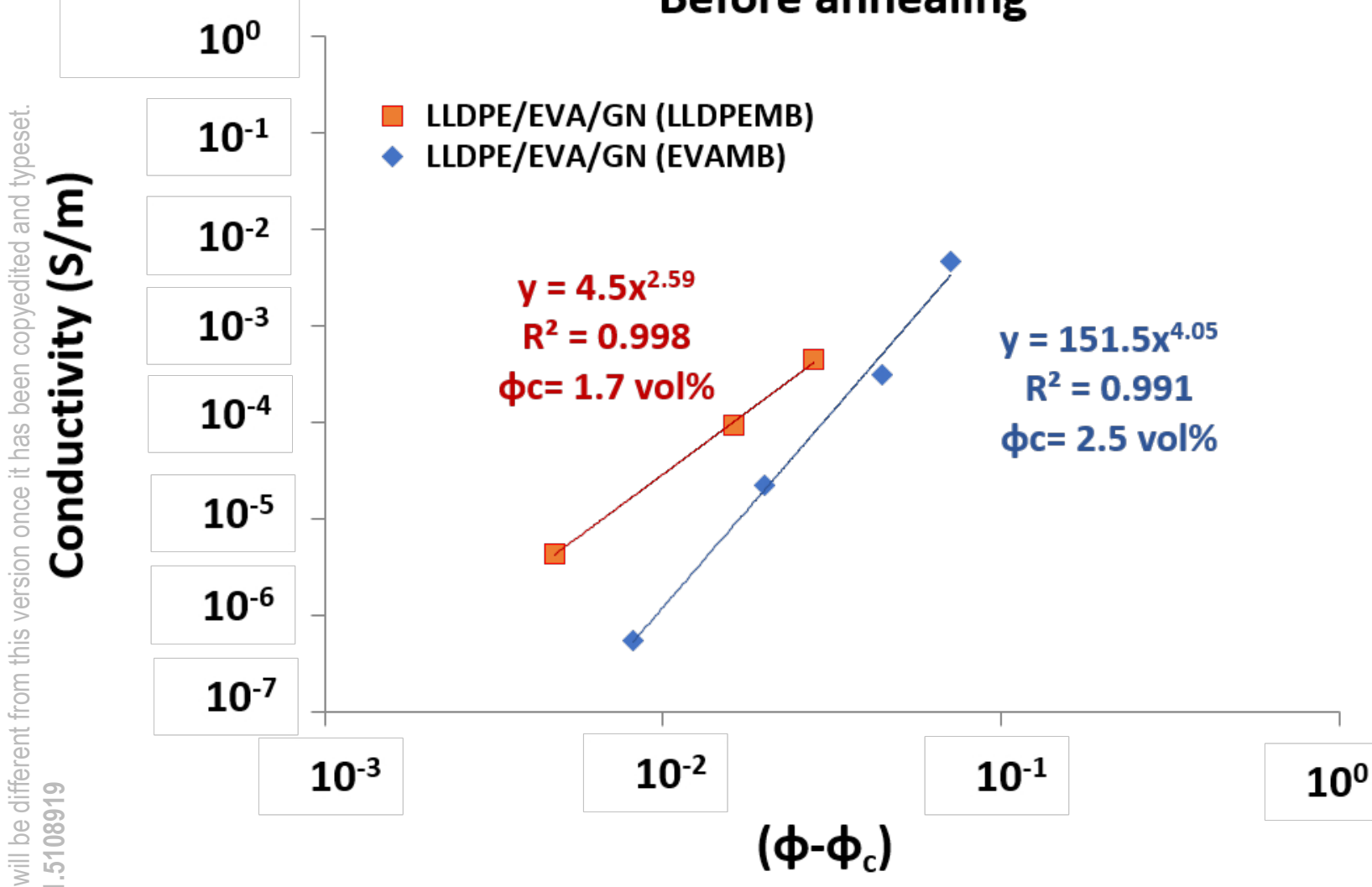




\section{After annealing}

\begin{tabular}{|c|c|}
\hline & $10^{-2}$ \\
\hline & $10^{-3}$ \\
\hline & $10^{-4}$ \\
\hline & $10^{-5}$ \\
\hline & $10^{-6}$ \\
\hline ○े & $10^{-7}$ \\
\hline & $10^{-8}$ \\
\hline ¿ & $10^{-9}$ \\
\hline
\end{tabular}

$10^{-4}$

- LLDPE/EVA/GN (LLDPEMB)

- LLDPE/EVA/GN (EVAMB) 